\title{
Energy transfer for high frequencies in built-up structures
}

\author{
A. LE BOT \\ Laboratoire de Tribologie et Dynamique des Systèmes CNRS \\ École centrale de Lyon, FRANCE alain.le-bot@ec-lyon.fr
}

\begin{abstract}
This paper is concerned with the prediction of vibrations at high frequencies in built-up structures. In the high frequency range, the dynamical behavior of structures is driven by energy transfer rather than modal aspects. The description of systems in terms of energy is thus well-suited and leads to closed-form equations of integral type on energy variables. The numerical solution of these equations is achieved with an appropriate software called CeReS, and the results are compared with experimental measurements on a multi-plate structure.
\end{abstract}

\section{Introduction}

The prediction of the dynamical behavior of stuctures in the low frequency range is a problem that is largely overcome today. Although there remains some difficulties, the numerous finite element software apply at least for the linear behavior and are used for the design of equipment. However, it is well-known that finite element analysis becomes rapidly inefficient as the frequency increases because of the unreasonable time-computation required.

In the transport industry, to improve the vibroacoustic comfort is an essential question that is of an increasing interest. But for the reason previously pointed out, the use of classical methods turns out to be limited to frequencies at the low end of the audible range. Methods with a lower numerical cost are then required. Of course, such a numerical gain is only possible in return to loss of information. Some simplifying assumptions are required. In this context, the Statistical Energy Analysis (SEA) has been developed for several decades [1]. Any complex structure is divided into simple connected subsystems. Based on a power balance for each subsystem, the Statistical Energy Analysis is concerned with the prediction of vibrational energy levels.

SEA is based on the restrictive assumption that vibrational fields are diffuse. Thus, a single degree of freedom is attached to subsystems i.e. the total vibrational energy or, alternatively, the modal energy. Alternative methods have been proposed to improve SEA and particularly to predict the repartition of vibrational energy inside subsystems. On that subject, let mention the Wave Intensity Analysis [2]. Fields are no longer assumed to be diffuse. More precisely, homogeneity is still assumed but intensity may be non isotropic. The directional dependance of intensity leads to a greater number of degrees of freedom than SEA but, in counterpart, a more accurate description of energy fields in subsystems. Another approach is found in the work of Nefske and Sung [3] where an analogy with the thermal conduction in material is developped. Vibrational fields may be neither homogeneous nor isotropic. This method is based on a local power balance and a local relationship relating energy flow with energy density analogous to Fourier's law in thermics. Nevertheless, 
the thermal conduction analogy also called Energy Finite Element Method $[4,5,6]$ or vibrational conductivity approach [7] is subject to some limitations that have been emphasized in literature $[7,8,9,10,11]$. One of these limitations is that the direct field predicted by this approach is like $1 / \sqrt{r}$ where $r$ is the source-receiver distance whereas the correct law is $1 / r$ for bidimensional systems like plates. In order to avoid this difficulty, this paper proposes a method rather based on an analogy with the radiative thermal transfer. This method significantly differs from the thermal conduction analogy. Some details are given in this text but for a complete comparison of both methods for circular plates, see Reference [9].

The first part of this text is intended for theoretical aspects of the method. Secondly, the underlying equations are numerically solved with the help of a software, called CeReS that has been specifically designed for application in vibroacoustics. Finally, two experiments on a built-up structure made of assembled plates were carried out and the results have been compared with the results of the numerical models provided by the software CeReS.

\section{General concepts}

The analysis of vibrating fields in the high frequency range requires us to consider several concepts which are discussed in this section.

\subsection{Traveling wave}

The most fundamental of these concepts is that any vibrating field may be viewed as a linear superposition of some traveling waves. The reasons of this choice instead of a modal decomposition will be highlighted throughout this text. But one can say here and now that standing waves can only exist at some particular frequencies, the eigenfrequencies, and are responsible for sudden variation of dynamical responses. This modal behavior is dominant in the low frequency range and the decomposition of vibrating field into stationary waves is well-suited. On the contrary, the higher is the frequency, the larger is the modal overlap and the less visible are the modes. The relevant phenomenon in the high frequency range is the energy transfer between any parts of the structure. In this matter, the underlying entity which explains in a convenient way these energy exchanges is the traveling wave rather than the stationary wave. This is why traveling waves are so important in the high frequency range.

We have not yet defined the high frequency domain. Several definitions appear in the related literature $[12,13]$. The main difficulty is that in the structural case wavenumbers and also wavelengths depend on the material properties. In the work of Wohlever and Bernhard [4], it has been remarked that results of energy models should be meant as a spatial average over a wavelength of 'exact' results of the equations of motion. Additional averaging processes should be introduced for an accurate interpretation of the macroscopic behavior predicted by energy models [1, 14]. But in this study, we adopt the point of view of Wohlever and Bernhard which is also the one of DeLanghe [14] and we define the high frequencies as the domain where at least several wavelengths lie in the system or, in other words, the characteristic length of the structure is much greater than the wavelength. When several subsystems are connected, the validity domain must be restricted. All subsystems must be separately in the high frequency band and thus high frequencies for the whole structure begin when several wavelengths lie in each subsystem. 
The first step in developing our model is to make an exhaustive list of all kinds of wave which can propagate inside the studied structure. The final experiment is carried out on a multiplate structure and we thus confine the present discussion to the case of vibrating plates. As we are concerned with some wavelengths which are much greater than the thickness of plates, the conventional theory of Love plates remains valid. It is well-known that three kinds of traveling waves may exist in an infinite extended Love plate. The first type is the bending wave responsible of out-of-plane motion. All quantities attached to this wave will be noted with a subscript $b$. The second and third types of wave are the longitudinal and transverse waves for in-plane motion with subscripts $l$ and $t$. The corresponding group velocities are denoted by $c_{\alpha}$ where $\alpha=b, l$ or $t$.

The fact that in the absence of curvature, all waves propagate independently of each other leads to a major simplification in describing vibrating field with a wave approach. No energy exchange takes place inside plates during propagation. The only way for a wave to exchange some energy with other kinds of wave is by reflection on a boundary. Of course this phenomenon usually called conversion mode must be taken into account in our model.

Special attention should be paid to the status of evanescent waves. These waves may exist in particular systems such as transverse vibrating beams and out-of-plane motion of plates. The question arising now is to decide whether the evanescent waves must be taken into account with the view of evaluating energy transfer in structures. Before giving an answer, let us make some remarks on the basis of the example of flexural waves in beams. An evanescent wave in beams gives rise to a transverse motion $v(x, t)=a e^{i \omega t-k_{b} x}$ where $k_{b}$ is the flexural wavenumber and $\omega$ is the circular frequency. The resulting time-averaged energy density is $W(x)=\rho|\partial v / \partial t|^{2} / 4+D\left|\partial^{2} v / \partial x^{2}\right|^{2} / 4=\rho \omega^{2} e^{-2 k_{b} x} / 2$ where $\rho$ is the mass per unit length and $D$ is the real-valued flexural rigidity of the beam. Thus the energy density decreases rapidly far from the origin. More exactly, the energy of an evanescent wave is negligible outside the vicinity of the singularity from which it emerges. On the other hand, the time-averaged energy flow is $I(x)=D \Re\left(\partial^{2} v / \partial x^{2} \times \partial^{2} v^{*} / \partial x \partial t-\partial^{3} v / \partial x^{3} \times \partial v^{*} / \partial t\right) / 2=0$ vanishes. Indeed, an evanescent wave is rarely alone and some cross-product terms appear in the evaluation of energy quantities when both traveling wave and evanescent wave exist. But there remains that in farfield evanescent wave are negligible and thus evanescent wave does not contribute to any large distance energy transfer. However, the presence of evanescent waves can modify drastically the behavior of the structure in the vicinity of singularities such as boundaries, interfaces or driving points. It could even arise that it dominates all other types of traveling wave. Consequently, we cannot be unaware of the presence of these waves. When a traveling wave of kind $\alpha$ impinges on a singularity, all other types of wave including evanescent one are reflected. It is then clear that the presence of evanescent waves acts deeply upon the mode conversion phenomenon. So, in what follows, the energy attached to evanescent waves will be systematically neglected when evaluating the vibrating energy inside the structure and the energy flow of these waves will not affect the energy balance at any point. But these waves must be accounted for evaluating the local energy efficiencies that drive energy exchange between traveling waves at connections of plates.

\subsection{Wave-packet}

Each time we shall be interested in transient aspects of the dynamical behavior of systems, the traveling wave description will turn out to be inappropriate. It appears that for the purpose of accounting for time-dependance while staying in the high frequency domain, the most natural generalization of traveling waves is the wave-packet concept. Wave-packet may be thought as a traveling wave of finite duration, or, in other words, a traveling wave amplitude-modulated by a 
pulse shaped waveform, which initially restricts the wave-packet to a finite spatial spread. The duration of the disturbance must be large compared with the period of the main oscillation such that the wave-packet comprises several cycles. The high frequency assumption then just states that the frequency $\omega$ of oscillation within the packet lies in the high frequency domain, that is the wavelength is smaller than a characteristic length of the system. In order that wave-packets behave like traveling waves during their passing, we must also add the assumption that the shape function varies slowly compared with the main oscillation. At initial instant, the Fourier transform of a wave-packet is a narrow band signal sharply peaked at $\omega$ with a bandwidth $\Delta \omega$ that is small relative to $\omega$. Recall that for a pure traveling wave, the spectrum is a delta Dirac function at $\omega$ while a pulse has a constant spectrum over the frequency domain or at least a wide-band spectrum for actual pulses.

In Appendix A, the case of gaussian wave-packets is studied. It has been shown that for both dispersive and non-dispersive media, initial gaussian wave-packets remain gaussian wave-packets during propagation. In addition to the fact that complete calculations can be carried out, this pleasant result justifies the choice of the gaussian shape for the study of a wave-packet example. It has been also shown that the frequency of the main oscillation remains unchanged during the propagation. This key result points out that if the high frequency assumption applies at initial time, it also applies at later time. Concerning the shape function, non-dispersive medium leads to a constant spatial spread whereas dispersive medium tends to increase the spatial spread as time goes on. Thus the assumption that time-variation induced by the shape function is slower than those due to the main oscillation is better verified.

Finally, it is well-known that wave-packets propagate with the group velocity $c$. Since the energy attached to the motion and the deformation of the medium vanishes outside the wave-packet, the group velocity is also the energy velocity.

\subsection{Some approximations and assumptions for high frequencies}

With these aspects in mind, we now turn to the description of the dynamical behavior of both traveling waves and wave-packets. All the material necessary in what follows may be summarized in three assumptions which will be applied throughout this text and which are set out now. These assumptions are rather common in the high frequency literature and we do not attempt to justify them from the study of exact solutions of the governing equation, wave equation or Love plate equation, for instance. Let remark that these assumptions, except the first one, only apply at high frequencies. They rather result from some approximations that will be specified.

The first assumption stems from the well-known Helmholtz-Kirchhoff formula in acoustics or other related formulae for more complicated waves, among them waves in plates or for more general situations that include time-dependance. The underlying idea of all these integral representation formulae is that at any point the vibrating field is the linear superposition of, on the one hand, the direct field emerging from actual sources and, on the other hand, the diffracted field or scattered field emerging from secondary sources located on boundary. It is not necessary to give all details about these representation formulae and in particular, the exact magnitude of secondary sources is of no importance for purposes which follow. The only relevant fact is that any vibrating field may be synthesized by summing traveling waves or wave-packets whose sources are clearly identified driving point, boundaries, interfaces and, more generally, any point where interaction of waves occurs. 
The second postulate states that all traveling waves and wave-packets are uncorrelated, that is the relative phase between an arbitrary pair of waves is a uniform random variable. In view of the first assumption, it should be distinguished between uncorrelation of actual sources and secondary sources. The first type is a physical assumption in the sense that it must be checked on the actual experiment that driving forces or moments are effectively uncorrelated. In opposition, uncorrelation of diffraction sources or mixed sources rather results from an approximation deliberately introduced in the description of deterministic systems. This approximation leads to neglect all interference effects in structures. This is a very important point for a complete understanding of the method. Modes cannot be predicted. We interpret the results of the method considered in this paper as averaged results in a local sense (spatial average over a wavelength, third octave band frequency average). Then spatial correlation within a wavelength are canceled but long-range correlation remains. This shortcoming may appear in the presence of some particular effects such as waveguide cut-off, block- and pass-band of periodic systems... These kinds of systems that require a specific treatment, are not considered in this paper.

Finally, the third and last assumption is the locality principle which may be expressed in these words. Any interaction process involving several waves interacting at a given point just depends on local geometry of system and wavefronts. In other words, local behavior depends on local properties. The most fundamental interest of the locality principle lies in the fact that it allows the substitution of any problem of interacting waves by a canonical problem with identical local geometry but extrapolated in such a manner that a closed-form solution is accessible. The simplest example for the use of the locality principle, is the reflection problem. The reflection coefficient of a plane wave impinging on an infinite plane may be used for any wave impinging on a regular boundary. Indeed, this is just an approximation valid at first order since the effects of curvature of both wavefront and boundary are not taken into account. But the locality principle states that the presence of remote obstacle is of no importance.

\subsection{Energy variables}

It has been pointed out that high frequency dynamics is governed by energy transfer rather than modal behavior. To this end, the choice of energy variables in place of kinematic variables such as pressure and velocity in acoustics and stress and displacement for structures, seems to be natural. In fact, it has been largely discussed in literature that the choice of energy variables may offer some significant advantages for high frequency modelling [1].

Thus, in this text, vibratory fields are fully described with the help of two energy variables, namely the energy density $W_{\alpha}(M, t)$, a scalar quantity and the energy flow $\mathbf{I}_{\alpha}(M, t)$, a vector quantity. Both depend on position $M$ and time $t$. By virtue of the uncorrelation assumption, all energy quantities are additive that is a linear superposition principle is valid for energy variables. This key result allows us to handle energy quantities in a very simple manner. In particular, energy variables have to be the sums of related energies attached to individual waves.

At any point $M$ and any time $t$, energy balance equation is:

$$
\operatorname{div} . \mathbf{I}_{\alpha}+p_{\alpha}^{\mathrm{diss}}+\frac{\partial W_{\alpha}}{\partial t}=\rho_{\alpha} \quad \alpha=b, l \text { or } t
$$

where $p_{\alpha}^{\text {diss }}$ is the power density being dissipated, $\rho_{\alpha}$ is the power density being injected by driving forces and $\partial W_{\alpha} / \partial t$ is the time-varying term of energy density. Of course power density being injected is assumed to be known, or at least, may be derived from imposed force or displacement with the use of asymptotic relationships commonly reported in the high frequency literature $[15,16]$. 
The plan is now to solve equation (1) for pure waves by evaluating all terms in the left-hand side and to deduce total energies $W_{\alpha}$ and $\mathbf{I}_{\alpha}$ by applying the linear superposition principle.

\section{Radiative energy transfer}

\subsection{Energy fields for pure waves}

In Appendix A of Reference [17], it is shown that cylindrical bending waves in plates verify two additional properties. First, the energy density is equally shared between kinetic energy $T_{\alpha}$ and potential energy or deformation energy $V_{\alpha}$.

$$
T_{\alpha}(M, t)=V_{\alpha}(M, t)
$$

Second, the energy flow magnitude is the energy density times group velocity.

$$
I_{\alpha}(M, t)=c_{\alpha} W_{\alpha}(M, t)
$$

A similar verification for in-plane cylindrical waves would give same result. It should be noted that two assumptions have been necessary to achieve this calculation, far-field hypothesis and evanescent wave neglecting. The last one has previously been justified. Since the near-field extends over few wavelengths, the far-field hypothesis is well suited in high frequencies where the wavelength is small. In Appendix A of this paper, the case of gaussian wave-packets is considered. It results in same conclusions.

In general systems, energy of waves should decay during propagation due to the action of damping phenomena. In the present text, we shall call damping all processes of conversion of the vibrational energy into a form of energy which is not taken into account, including heat, sound and so on. The nature of physical processes responsible of dissipation, in sense of the above definition, may be of various kinds. Viscous forces applied to a structure by a surrounding fluid, internal friction forces usually taken into account in Hooke's law by substituting a complex elastic modulus, friction at interfaces of metal sheets of built-up structures, acoustic radiation of boundaries are some examples of such processes. However, among this wide variety of aspects, two classes may be discerned, damping phenomena occuring within the components of the system which are included in the term $p_{\alpha}^{\text {diss }}$ and other phenomena for which the dissipation is confined to the neighborhood of interfaces or boundaries and which will be taken into account later when considering the problem of reflection at boundaries. Among the former, viscous forces result in a power density being dissipated which is proportional to local kinetic energy. In opposition, a complex elastic modulus leads to an energy loss proportional to potential energy. It has previously been remarked that kinetic and potential components of energy are equal for a pure wave, so that both damping laws match. In general, equality of both forms of energy remains valid for a superposition of waves by virtue of the linear superposition principle. With these considerations in mind, we adopt a universal damping law for internal losses. A wave of energy flow magnitude $I_{\alpha}$, after traversing a thickness $d s$ in its direction of propagation, will be weakened in such a way that $d I_{\alpha}=\eta \omega W_{\alpha} d s$ where $\omega$ is the circular frequency of the wave and $\eta$ an appropriate damping loss factor. Sometimes, it will be more convenient to use a rather acoustical notation by introducing an absorption factor $m$ defined by $d I_{\alpha}=m c_{\alpha} W_{\alpha} d s$. The power density being dissipated is then related to the energy density by:

$$
p_{\alpha}^{\text {diss }}(M, t)=\eta \omega W_{\alpha}(M, t)=m c_{\alpha} W_{\alpha}(M, t)
$$


Let us return to our first aim which was to calculate energy fields for pure waves. The present method requires the knowledge of the direct field, that is the energy density created by a point source in an infinitely extended medium. This energy density is noted $G_{\alpha}(S, \tau ; M, t)$ where $S$ is the source point sending up a signal at time $\tau$ and $M$ is the observation point at time $t$. The related energy flow is noted $\mathbf{H}_{\alpha}(S, \tau ; M, t)$. Sometimes, we shall use the notation $H_{\alpha}$ for the magnitude of the energy flow vector. The power balance to be verified is:

$$
\operatorname{div}_{M} . \mathbf{H}_{\alpha}(S, \tau ; M, t)+m c_{\alpha} G_{\alpha}(S, \tau ; M, t)+\frac{\partial G_{\alpha}}{\partial t}(S, \tau ; M, t)=\delta_{S}(M) \delta_{\tau}(t)
$$

for an impulse excitation. Recall that $G_{\alpha}$ and $H_{\alpha}$ must be related by the relationship (3). In Appendix B of this text, the outgoing solution of (3) and (5) is found to be:

$$
\begin{aligned}
G_{\alpha}(S, \tau ; M, t) & =G_{\alpha}(S, M) \delta\left(t-\tau-S M / c_{\alpha}\right) \\
\mathbf{H}_{\alpha}(S, \tau ; M, t) & =\mathbf{H}_{\alpha}(S, M) \delta\left(t-\tau-S M / c_{\alpha}\right)
\end{aligned}
$$

where the steady state solutions $G_{\alpha}(S ; M)$ and $\mathbf{H}_{\alpha}(S ; M)$ have been introduced (see also Appendix B of $[17])$

$$
\begin{aligned}
G_{\alpha}(S, M) & =\frac{e^{-m S M}}{\gamma_{0} c_{\alpha} S M^{n-1}} \\
\mathbf{H}_{\alpha}(S, M) & =c_{\alpha} G_{\alpha}(S, M) \mathbf{u}_{S M}
\end{aligned}
$$

where $\gamma_{0}$ is the solid angle of space of dimension $n=1,2$ or 3 and $\mathbf{u}_{S M}$ the unit vector from $S$ toward $M$. Note that these expessions have been derived under the assumption that $m$ and $c_{\alpha}$ are constant or, in other words, that the space of propagation is homogeneous and isotropic.

\subsection{Complete energy fields}

By virtue of linearity and the decomposition into direct and diffracted fields, the case of complete wave fields is handle by adding direct fields emerging from primary sources $\rho_{\alpha}(S, \tau)$ and from secondary sources that will be noted $\sigma_{\alpha}(P, \mathbf{u}, \tau)$ where $P$ belongs to the boundary $\partial \Omega$, $\mathbf{u}$ a direction and $\tau$ the time. It results in:

$$
\begin{gathered}
W_{\alpha}(M, t)=\int_{-\infty}^{\infty} \int_{\Omega} \rho_{\alpha}(S, \tau) G_{\alpha}(S, \tau ; M, t) d S d \tau+ \\
\int_{-\infty}^{\infty} \int_{\partial \Omega} \sigma_{\alpha}\left(P, \mathbf{u}_{P M}, \tau\right) G_{\alpha}(P, \tau ; M, t) d P d \tau \\
W_{\alpha}(M, t)=\int_{\Omega} \rho_{\alpha}\left(S, t-S M / c_{\alpha}\right) G_{\alpha}(S, M) d S+\int_{\partial \Omega} \sigma_{\alpha}\left(P, \mathbf{u}_{P M}, t-P M / c_{\alpha}\right) G_{\alpha}(P ; M) d P
\end{gathered}
$$

A similar relationship is obtained for energy flow:

$$
\mathbf{I}_{\alpha}(M, t)=\int_{\Omega} \rho_{\alpha}\left(S, t-S M / c_{\alpha}\right) \mathbf{H}_{\alpha}(S, M) d S+\int_{\partial \Omega} \sigma_{\alpha}\left(P, \mathbf{u}_{P M}, t-P M / c_{\alpha}\right) \mathbf{H}_{\alpha}(P, M) d P
$$

Of course primary sources $\rho_{\alpha}$ are assumed to be known but the secondary sources $\sigma_{\alpha}$ remain unknown and an additional equation has to be sought to determine them.

\subsection{Radiative intensity}

In order to describe the directional dependance of the energy flow, it will be convenient to introduce the radiative intensity defined as the energy flow per unit solid angle and unit area normal to the 


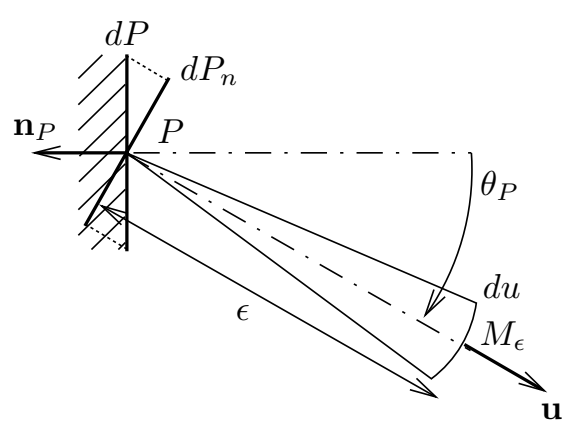

Figure 1: Radiative intensity emanating from an infinitesimal surface $d P$ into solid angle $d u$ about u.

rays. It will be denoted by $I_{\alpha}(M, \mathbf{u}, t)$ which depends on the direction $\mathbf{u}$. At any point $M$ and any time $t$, the energy flow $\mathbf{I}_{\alpha}(M, t)$ is obtained by summing the radiative intensity over all solid angles.

$$
\mathbf{I}_{\alpha}(M, t)=\int I_{\alpha}(M, \mathbf{u}, t) \mathbf{u} d u
$$

Radiative intensity on the boundary $\partial \Omega$ may be related to the secondary source magnitude $\sigma_{\alpha}$. In this matter, let consider an infinitesimal surface $d P$ of the boundary. The radiative intensity in the direction $\mathbf{u}$ is given by

$$
I_{\alpha}(P, \mathbf{u}, t)=\frac{d \mathcal{P}_{\alpha}}{d P_{n} d u}
$$

where $d P_{n}=d P \cos \theta_{P}$ is the normal surface and $d \mathcal{P}_{\alpha}$ the power into the solid angle $d u$ (see Figure $1)$. The energy flow emanates from a secondary source whose magnitude is $\sigma_{\alpha} d P$, then

$$
I_{\alpha}(P, \mathbf{u}, t)=\lim _{\epsilon \rightarrow 0} \sigma_{\alpha}\left(P, \mathbf{u}, t-\epsilon / c_{\alpha}\right) d P \frac{H_{\alpha}\left(P, M_{\epsilon}\right) \epsilon^{n-1} d u}{d P_{n} d u}=\frac{\sigma_{\alpha}(P, \mathbf{u}, t)}{\gamma_{0} \cos \theta_{P}}
$$

where $M_{\epsilon}$ is a point at a small distance $\epsilon$ from $P$ in the direction $\mathbf{u}$. Thus, this relationship implies that the use of the radiative intensity $I_{\alpha}(P, \mathbf{u}, t)$, that is the energy flow per unit solid angle and area normal to the rays, or the use of the directional emitted flux $\sigma_{\alpha}(P, \mathbf{u}, t) / \gamma_{0}$ that is the energy flow per solid angle and area, as unknown for the reflection problem, is a purely formal question. The source $d P$ is said to be diffuse when the radiative intensity is constant or, in other words, when the directional emitted flux varies with the cosine of the polar angle.

The radiative intensity is not only defined in the vicinity of the boundary but also inside the domain itself. Consider a point $M$ inside $\Omega$ and a direction $\mathbf{v}$. The radiative intensity $I_{\beta}(M, \mathbf{v}, t)$ attached to a wave $\beta$ is originated from the source $\sigma_{\beta}$ located at $Q$ on the boundary in view of $M$ and secondly from all actual sources $\rho_{\beta}$ belonging between $Q$ and $M$. Explicitly,

$$
I_{\beta}(M, \mathbf{v}, t) \mathbf{v} d v=\int_{(M, d v)} \rho_{\beta}\left(S, t-S M / c_{\beta}\right) \mathbf{H}_{\beta}(S, M) d S+\sigma_{\beta}\left(Q, \mathbf{v}, t-Q M / c_{\beta}\right) \mathbf{H}_{\beta}(Q, M) d Q
$$

where the first term of the right-hand side is an integral over the cone $(M, d v)$ of vertex $M$ and angle $d v$ about $\mathbf{v}$. The second term is the contribution of the sources that belong on $d Q$ (see Figure 2 ). Of course by virtue of (13), the expression (12) is recovered by integrating (16) over all solid angles. 


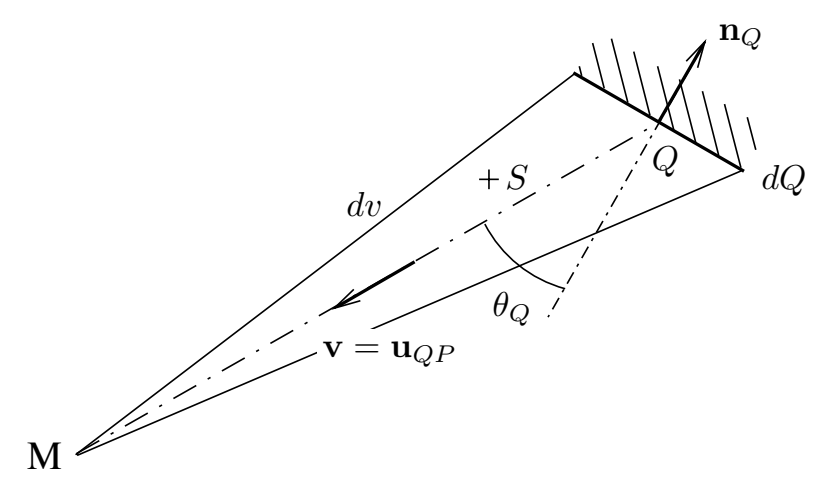

Figure 2: Radiative intensity at any point $M$ inside the domain $\Omega$. Both actual sources $S$ located inside the cone $(M, d v)$ and the boundary source $d Q$ contributes.

On the other hand, the incident flux at any point $P$ of the boundary stemming from $\mathbf{v}$ is readily found by applying (16).

$$
\begin{aligned}
I_{\beta}(P, \mathbf{v}, t) \mathbf{v} \cdot \mathbf{n}_{P} d v= & {\left[\int_{\left(P, \mathcal{Q}_{\beta} \beta\right.}\left(S, t-S P / c_{\beta}\right) H_{\beta}(S, P) \mathbf{v} d S+\right.} \\
& \left.\sigma_{\beta}\left(Q, \mathbf{v}, t-Q P / c_{\beta}\right) H_{\beta}(Q, P) \mathbf{v} d Q\right] . \mathbf{n}_{P}
\end{aligned}
$$

Notations are defined on Figure 3.

\subsection{Reflection at boundaries}

The required equation for $\sigma_{\alpha}$ is found by applying the power balance at any point $P$ on the boundary $\partial \Omega$. To this end, the bidirectional reflectivity of the boundary is introduced. The bidirectional reflectivity $R_{\beta \alpha}(\mathbf{v}, \mathbf{u})$ at any point $\mathrm{P}$ is defined as the part of the radiative intensity in the direction $\mathbf{u}$ attached to the wave $\alpha$, induced by a unit incident flux of the wave $\beta$ stemming from the direction $\mathbf{v}$ (see Figure 4). The bidirectional reflectivity depends on two directions. The total radiative intensity $I_{\alpha}(P, \mathbf{u}, t)$ is obtained by summing all the contributions of directions $\mathbf{v}$ and types $\beta$ of wave. So,

$$
I_{\alpha}(P, \mathbf{u}, t)=\sum_{\beta} \int R_{\beta \alpha}(\mathbf{v}, \mathbf{u}) I_{\beta}(P, \mathbf{v}, t) \mathbf{v} \cdot \mathbf{n}_{P} d v
$$

where $I_{\beta}(P, \mathbf{v}, t) \mathbf{v} \cdot \mathbf{n}_{P}$ is the incident flux per unit solid angle of a wave $\beta$ from the incident direction $\mathbf{v}$. The integration runs over all incident solid angles. Our purpose is now to explain the different terms occuring in this relationship.

The radiative intensity of the surface $d P$ in the direction $\mathbf{u}$ is given by (15). On, the other hand, the incident flux at $P$ stemming from $\mathbf{v}$ is given by (17). Then, the reflection condition (18) reads:

$$
\begin{aligned}
\frac{\sigma_{\alpha}(P, \mathbf{u}, t)}{\gamma_{0} \cos \theta_{P}}=\sum_{\beta} \quad & {\left[\int_{\Omega} R_{\beta \alpha}\left(\mathbf{u}_{S P}, \mathbf{u}\right) \rho_{\beta}\left(S, t-S P / c_{\beta}\right) \mathbf{H}_{\beta}(S, P) d S+\right.} \\
& \left.\int_{\partial \Omega} R_{\beta \alpha}\left(\mathbf{u}_{Q P}, \mathbf{u}\right) \sigma_{\beta}\left(Q, \mathbf{u}_{Q P}, t-Q P / c_{\beta}\right) \mathbf{H}_{\beta}(Q, P) d Q\right] . \mathbf{n}_{P}
\end{aligned}
$$

This integral equation fully determines the unknowns $\sigma_{\alpha}$. 


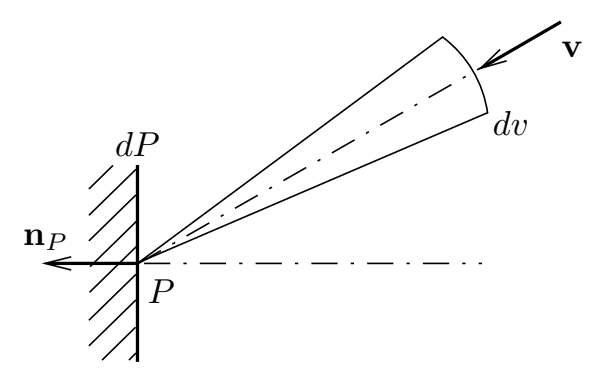

Figure 3: Incident flux at a point $P$ of the boundary stemming from the solid angle $d v$ about $\mathbf{v}$.

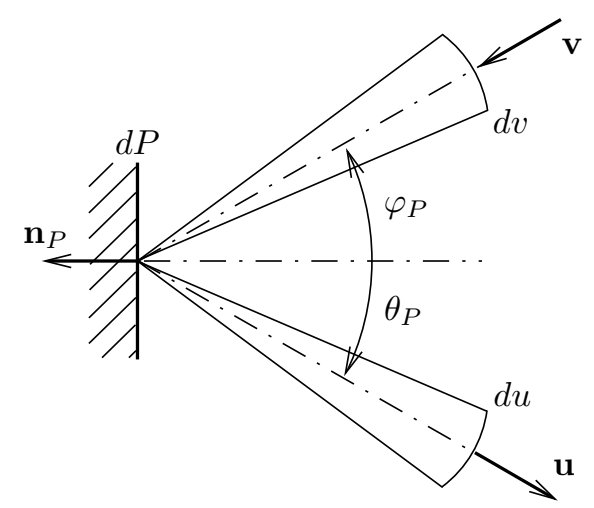

Figure 4: The bidirectional reflectivity depends on an incident direction $\mathbf{u}$ and a reflected direction v. 


\begin{tabular}{|c||c|c|c|}
\hline & $n=1$ & $n=2$ & $n=3$ \\
\hline \hline$\gamma_{0}$ & 2 & $2 \pi$ & $4 \pi$ \\
\hline$\gamma$ & 1 & 2 & $\pi$ \\
\hline
\end{tabular}

Table 1: Solid angle $\gamma_{0}$ of space and value of the constant $\gamma$ versus the space dimension $n$.

We define the directional hemispherical reflectivity $R_{\beta \alpha}(\mathbf{v})$ of a surface $d P$ as the total reflected energy flux leaving $d P$ into all directions due to the directional incident flux $I(P, \mathbf{v}) \cos \theta_{P}$.

$$
R_{\beta \alpha}(\mathbf{v})=\int R_{\beta \alpha}(\mathbf{v}, \mathbf{u}) \cos \theta_{P} d u
$$

where the integral runs over all reflected directions. The directional reflectivity is sometimes called reflection efficiency. This is the ratio of the reflected power of the wave $\alpha$ over the incident power of the wave $\beta$ and, thus, its value runs from 0 to 1 .

We now consider the case of perfectly diffuse reflection. A perfect diffuse reflector also called Lambert's reflector has a bidirectional reflectivity which is u-independant. For such reflectors, the directional reflectivity is:

$$
R_{\beta \alpha}(\mathbf{v})=R_{\beta \alpha}\left(\mathbf{v}, \mathbf{u}_{0}\right) \int \cos \theta_{P} d u=\gamma R_{\beta \alpha}\left(\mathbf{v}, \mathbf{u}_{0}\right)
$$

where $\mathbf{u}_{0}$ is any fixed reflected direction. The values of the constant $\gamma$ for different dimensions are summarized in Table 1.

In the perfectly diffuse case, the right-hand side of the reflection law (18) does not depend on the direction $\mathbf{u}$ and so the radiative intensity from a diffuse reflector is constant over all directions u. The relationship (15) then leads to a directional emitted flux $\sigma_{\alpha}(P, \mathbf{u}, t) / \gamma_{0}$ which varies with the cosine of the polar angle $\theta_{P}$. We then write:

$$
\sigma_{\alpha}(P, \mathbf{u}, t)=\sigma_{\alpha}(P, t) \cos \theta_{P}
$$

This is the Lambert's law.

By substituting (21) and (22) into the integral equation (19), we obtain a new integral equation on $\sigma_{\alpha}(P, t)$ for the particular case of diffuse reflection.

$$
\begin{aligned}
\frac{\gamma}{\gamma_{0}} \sigma_{\alpha}(P, t)=\sum_{\beta} \quad & {\left[\int_{\Omega} R_{\beta \alpha}\left(\mathbf{u}_{S P}\right) \rho_{\beta}\left(S, t-S P / c_{\beta}\right) \mathbf{H}_{\beta}(S, P) d S+\right.} \\
& \left.\int_{\partial \Omega} R_{\beta \alpha}\left(\mathbf{u}_{Q P}\right) \sigma_{\beta}\left(Q, t-Q P / c_{\beta}\right) \cos \theta_{Q} \mathbf{H}_{\beta}(Q, P) d Q\right] . \mathbf{n}_{P}
\end{aligned}
$$

In acoustics, just one type of wave may propagate in the fluid. Reflection from materials is rather characterized in terms of the absorptivity $\alpha$ or absorption factor which is related to the reflectivity with $\alpha=1-R$. Its value is usually evaluated by introducing a piece of material into a reverberent room and by measuring the resulting reverberation time. Since a diffuse field stays in the room, the absorption factor then determined is an averaged value over all incident directions of the directional absorptivity. It should be noticed that the directional absorptivity can be measured for any incident direction with a Kunt's tube. Unfortunately, these values are rarely available in literature for usual material. Anyway, it is a common assumption in room acoustics that the absorption factor does not depend on the incident direction $\mathbf{v}$. The integral equation (23) then reduces to:

$$
\frac{\sigma(P, t)}{4}=(1-\alpha)\left[\int_{\Omega} \rho(S, t-S P / c) \mathbf{H}(S, P) d S+\int_{\partial \Omega} \sigma(Q, t-Q P / c) \cos \theta_{Q} \mathbf{H}(Q, P) d Q\right] . \mathbf{n}_{P}
$$


Recall that $\gamma_{0} / \gamma=4$ for 3-dimensional space. This integral equation has been studied in Reference [18].

\subsection{Transmission at interfaces}

The analysis of reflection and transmission at the interface between two media or more, is relatively straightforward, following a similar way as in the previous section. All quantities are labelled with a subscript $i$ referring to the medium that is considered. For instance, at any point $P$ belonging on the interface, $\sigma_{i, \alpha}$ denotes the directional emitted flux towards the medium $i$.

Now the reflection condition (18) is replaced by some transmission conditions. It is tacitely assumed that reflection is the particular case of transmission from a system to itself. Thus, the transmission conditions read:

$$
I_{i, \alpha}(P, \mathbf{u}, t)=\sum_{j, \beta} \int R_{j i, \beta \alpha}(\mathbf{v}, \mathbf{u}) I_{j, \beta}(P, \mathbf{v}, t) \mathbf{v} \cdot \mathbf{n}_{P} d v
$$

There are as many equations as types $\alpha$ of wave and systems $i$ connected at $P$. Further, we expand the different terms in the same way as for the reflection condition in the previous section. This yields:

$$
\begin{aligned}
\frac{\sigma_{i, \alpha}(P, \mathbf{u}, t)}{\gamma_{0} \cos \theta_{P}}=\sum_{j, \beta} & {\left[\int_{\Omega_{j}} R_{j i, \beta \alpha}\left(\mathbf{u}_{S P}, \mathbf{u}\right) \rho_{j, \beta}\left(S, t-S P / c_{\beta}\right) \mathbf{H}_{j, \beta}(S, P) d S+\right.} \\
& \left.\int_{\partial \Omega_{j}} R_{j i, \beta \alpha}\left(\mathbf{u}_{Q P}, \mathbf{u}\right) \sigma_{j, \beta}\left(Q, \mathbf{u}_{Q P}, t-Q P / c_{\beta}\right) \mathbf{H}_{j, \beta}(Q, P) d Q\right] . \mathbf{n}_{P}
\end{aligned}
$$

This set of integral equations fully determines the unknowns $\sigma_{i, \alpha}$.

The case of diffuse transmission is reached by substituting the Lambert's law (22) into the set of integral equations. We readily obtain:

$$
\begin{aligned}
\frac{\gamma}{\gamma_{0}} \sigma_{i, \alpha}(P, t)=\sum_{j, \beta} \quad & {\left[\int_{\Omega_{j}} R_{j i, \beta \alpha}\left(\mathbf{u}_{S P}\right) \rho_{j, \beta}\left(S, t-S P / c_{\beta}\right) \mathbf{H}_{j, \beta}(S, P) d S+\right.} \\
& \left.\int_{\partial \Omega_{j}} R_{j i, \beta \alpha}\left(\mathbf{u}_{Q P}\right) \sigma_{j, \beta}\left(Q, \mathbf{u}_{Q P}, t-Q P / c_{\beta}\right) \mathbf{H}_{j, \beta}(Q, P) d Q\right] . \mathbf{n}_{P}
\end{aligned}
$$

This is the set of integral equations corresponding to diffuse transmission. This set has been derived in Reference [17] for two plates in steady state condition for a single type of wave.

\subsection{Non-convex domain}

Until now, we have tacily assumed that the domain of energy propagation is convex. However, such an assumption is no longer necessary.

We must consider that the energy emanating from an actual source $S$ or a boundary source $Q$ cannot reach a point $M$ if an obstacle is encountered in its path. In such a case, the energy is reflected and/or absorbed by the obstacle. This secondary emission of energy is accounted for by putting a boundary source on the obstacle.

Thus, in the relationships (11) and (12) must be modified in such a way that only the sources $S$ and $Q$ visible by the point $M$ have to be accounted for (see Figure 5). In the same way, when integrating (18) over all solid angles $d v$, only the sources visible by $P$ can contribute to the incident flux at $P$. The relationships $(19,23,26,27)$ are then modified. 


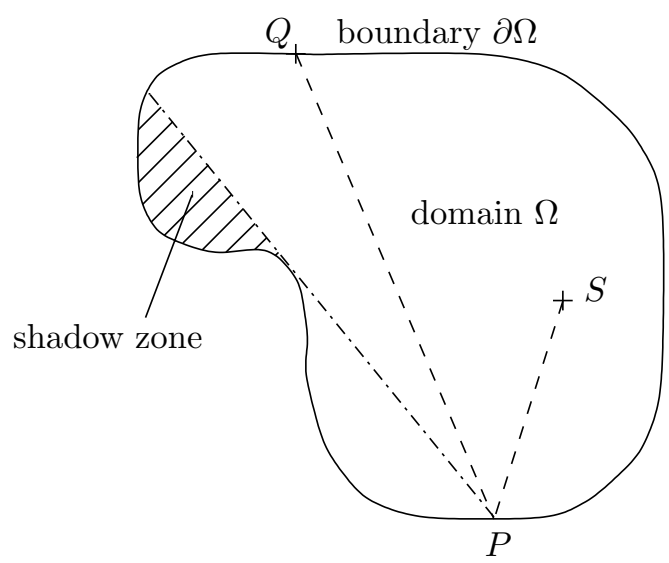

Figure 5: Non convex domain. The point $P$ is illuminated by sources $Q$ and $S$ respectively located on the boundary $\partial \Omega$ and in the domain $\Omega$ except the shadow zone.

A simple way to discard these hidden sources is to substitute the expressions $(8,9)$ of direct fields for the following new values:

$$
\begin{aligned}
G_{\alpha}(S, M) & =\frac{e^{-m S M}}{\gamma_{0} c_{\alpha} S M^{n-1}} V(S, M) \\
\mathbf{H}_{\alpha}(S, M) & =c_{\alpha} G_{\alpha}(S ; M) \mathbf{u}_{S M}
\end{aligned}
$$

where $V(S, M)$ is the visibility function which value is one when $S$ is visible from $M$ and zero otherwise.

\section{One-dimensional systems}

The particular case of one-dimensional systems is discussed in this section. For the sake of simplicity, we further assume that only a single wave can propagate in the system. For such a simple system, the boundary is composed of two extremities. Let denote by $s$ the space variable along the system, the origin is chosen in such a manner that the extremities are located at $s=0$ and $s=l$. Now, if the power supplied to the system is coming from these extremities (that is, no driving point is located inside the system), the relationship (11) for the energy density $W$ reduces to:

$$
W(s, t)=\frac{1}{2} \sigma^{+}(t-s / c) e^{-m s}+\frac{1}{2} \sigma^{-}(t-(l-s) / c) e^{-m(l-s)}
$$

where $\sigma^{+} / 2$ and $\sigma^{-} / 2$ are the emmited power by respectively the left and the right extremities. Deriving the expression (30) with respect to time and space variables gives:

$$
\begin{aligned}
2 W & =\sigma^{+} e^{-m s}+\sigma^{-} e^{-m(l-s)} \\
2 \partial_{t} W & =\sigma^{{ }^{\prime}} e^{-m s}+\sigma^{{ }^{\prime}} e^{-m(l-s)} \\
2 \partial_{t}^{2} W & =\sigma^{+^{\prime \prime}} e^{-m s}+\sigma^{-^{\prime \prime}} e^{-m(l-s)} \\
2 \partial_{s}^{2} W & =\left(\frac{\sigma^{+^{\prime \prime}}}{c^{2}}+\frac{2 m}{c}{\sigma^{+}}^{\prime}+m^{2} \sigma^{+}\right) e^{-m s}+\left(\frac{\sigma^{+^{\prime \prime}}}{c^{2}}+\frac{2 m}{c}{\sigma^{+}}^{\prime}+m^{2} \sigma^{+}\right) e^{-m(l-s)}
\end{aligned}
$$


By combining these equalities, it is straigthforward to check that the energy density $W$ verifies the partial differential equation:

$$
\frac{\partial^{2} W}{\partial s^{2}}(s, t)-\frac{1}{c^{2}} \frac{\partial^{2} W}{\partial t^{2}}(s, t)-\frac{2 m}{c} \frac{\partial W}{\partial t}(s, t)-m^{2} W(s, t)=0
$$

which is exactly the equation derived in Reference [11] from another point of view. In Reference [11], this equation is discussed and compared with another equation which has been proposed for the energy density [3]. The latter equation is based on the assumption that the energy flows like the thermal flows in material. This leads to an analogy with a heat conduction problem. But the telegraph type equation (35) rather suggests that a more correct analogy should be with a radiative heat transfer problem. This is the purpose of the next section.

\section{$5 \quad$ Analogy with radiative heat transfer}

A glance at any radiative heat transfer treatise reveals that the vibrational energy as considered in this paper travels the same way as radiative heat. We will discuss here the similarities and dissimilarities of both problems.

At first, we did consider that vibrating energy decreases during its travel. Such a phenomemon does not exist for the propagation of heat in vacuum or in air but appears when considering a semitransparent medium. This is the Beer's law. But generally this heat absorption immediatly goes with an emission of the same amount of energy. Unlike, in this work, the vibrational energy being dissipated is considered as lost or, more exactly, is transformed into internal energy that cannot reappear in a vibratory form.

We saw that in plates, three types of wave can travel. On the contrary, heat propagates with a unique speed that is the velocity of electromagnetic waves in the medium at hand. In the structural case, this difference leads to more rich models. Obviously, in the acoustical case, this difference vanishes.

Reflection of energy on boundaries highlights a more fundamental difference. When considering absorbing walls that is for a reflection efficiency less than one, the dissipated energy is lost for the vibrating system. However, in thermics, this energy raises the wall temperature. Following Stefan's law, this wall radiates some energy. The emissivity of a wall is equal to the absorptivity, this is the Kirchhoff's law. No energy is lost. In fact, in thermics, one is rather interested in the temperature of bodies submitted to the radiation. These bodies may be considered as being located on the boundary of the domain of thermal propagation. On the other hand, in mechanics, one usually seek to estimate the vibrational energy inside the structures. This is for this reason that we began this paper by deriving the relationships $(11,12)$ for the energy quantities inside the domain. This is a real difference of scientific strategy.

Despite this differences, there is a possibility to apply thermal sofware dealing with radiative transfer to mechanical or acoustical problems. It seems that this possibility has never been emphasized in literature. The numerous relationships for view factors available in thermal books are certainly re-usable in mechanics. For practical reasons, we prefered to design a specific software for acoustics and mechanics. We will briefly describe it in the next section. 


\section{Description $\mathrm{f}$ the software CeReS}

The software CeReS has been designed to solve the equations $(23,27)$ for a limited number of cases. In Reference [18], results for acoustical enclosures have been compared with results of a ray-tracing software for steady state conditions. It results in a good agreement. In this text, we are rather interested in structures made of assembled plates.

Each plate is defined as a part of plane surrounded by a polygonal line. The plates are convex or not. These plates are joined by their edges. The joints may be composed of an arbitrary number of plates. The geometry of the plates as well as the constitution of the joints must be specified to the software in a special data file. The structure then obtained may be subjected to point loadings. These sources are described in terms of their positions $S_{s}, s=1,2 \ldots$ and the powers $\rho_{\alpha}^{s}$ being injected into any kind $\alpha$ of waves. The response may be computed at any point $M$ of any plate.

The damping occurs in two ways. On the one hand, a damping loss factor $\eta$ is attached to each plate. It is responsible of the decrease of energy during propagation. On the other hand, an absorption factor $\alpha$ is attached to each edge of each plate. It is responsible of the absorption of the energy when waves impinge on the boundary. In the software CeReS, the reflection efficiencies $R_{\beta \alpha}$ are evaluated on the basis of the equilibrium of forces and moments at the interface as well as the continuity of displacements. For free edge, clamped edge and simply supported edge, the calculation of these efficiencies is done in Appendix $\mathrm{C}$ and for general joint composed of an arbitrary number of plates, see Reference [19]. These considerations leads to non-dissipative reflection efficiencies, that is the sum equals unity. To take into account extra-damping which may occur at edges or interfaces, the user can specify the absorption factor $\alpha$ for each edge. Reflection efficiencies theoretically predicted are then multiplied by this factor $\alpha$.

The software CeReS solves the equations $(23,27)$ for steady state conditions. In this matter, a boundary element method is applied. Each edge of plates is divided into a limited number of elements $L_{k}, k=1,2 \ldots$ of equal size. The magnitudes $\sigma_{\alpha}$ of the boundary sources are assumed to be constant over each boundary element. Thus, three unknowns $\sigma_{\alpha}^{k}, \alpha=b, l$ or $t$ are attached to the boundary element numbered $k$. For each element $k$, equation (23) or (27), depending on the position of the element at edge or interface, is applied at the middle $P_{k}$ of the element. This point $P_{k}$ is called the collocation point. First, for an element $k$ located at edge

$$
\sigma_{\alpha}^{k}=\pi\left[\sum_{\beta, s} \rho_{\beta}^{s} R_{\beta \alpha} H_{\beta}\left(S_{s}, P_{k}\right) \cos \theta_{P_{k}}+\sum_{\beta, l} \sigma_{\beta}^{l} \int_{L_{l}} R_{\beta \alpha} H_{\beta}\left(Q, P_{k}\right) \cos \theta_{Q} \cos \theta_{P_{k}} d Q\right]
$$

where $\theta_{P_{k}}$ is the incidence angle at the collocation point $P_{k}, \theta_{Q}$ the emanating direction, $R_{\beta, \alpha}$ is evaluated for the incidence angle. Second, for an element located at interface,

$$
\sigma_{i, \alpha}^{k}=\pi\left[\sum_{j, \beta, s} \rho_{j, \beta}^{s} R_{j i, \beta \alpha} H_{j, \beta}\left(S_{s}, P_{k}\right) \cos \theta_{P_{k}}+\sum_{j, \beta, l} \sigma_{\beta}^{l} \int_{L_{l}} R_{j i, \beta \alpha} H_{j, \beta}\left(Q, P_{k}\right) \cos \theta_{Q} \cos \theta_{P_{k}} d Q\right]
$$

where the sum runs over all connected plates, wave types and sources.

Thus, the set of equations $(36,37)$ leads to a system of linear equations for the unknowns $\sigma_{\alpha}^{k}$. The coefficients involve some integrals evaluated by Gauss quadrature. It should be pointed out that these integrals are regular, unlike the singular integrals involved in the classical boundary element method, allowing a fast and accurate computation. This linear system is solved with a Lapack [20] routine. Once the source magnitudes $\sigma_{\alpha}^{k}$ are computed, the energy density or energy 
flow inside each plate is evaluated from the equations $(38,39)$.

$$
W_{\alpha}(M)=\sum_{s} \rho_{\alpha}^{s} G_{\alpha}\left(S_{s}, M\right)+\sum_{k} \sigma_{\alpha}^{k} \int_{L_{k}} G_{\alpha}(P, M) \cos \theta_{P} d P
$$

for energy density at $M$ and

$$
\mathbf{I}_{\alpha}(M)=\sum_{s} \rho_{\alpha}^{s} \mathbf{H}_{\alpha}\left(S_{s}, M\right)+\sum_{k} \sigma_{\alpha}^{k} \int_{L_{k}} \mathbf{H}_{\alpha}(P, M) \cos \theta_{P} d P
$$

for energy flow. In these expressions, the sums of the right-hand side run over all sources acting on the plate where $M$ lies.

\section{$7 \quad$ SEA for assembled plates}

Statistical Energy Analysis is well-suited for built-up structures in high frequency range. It may be found many variants in the literature. The structure is first subdivided into several subsystems. We are concerned with assembled plates and the most natural choice for these subsystems is that each plate and each wave type is one subsystem. For the sake of simplicity, we introduce the double subscript $k=i, \alpha$ for the plate $i$ and wave $\alpha$. Let denote by $\bar{W}_{k} A_{k}$ the total vibrational energy contained within the subsystem $k=i, \alpha$ with $A_{k}$ being the area of plate $i$ and $\bar{W}_{k}$ the mean vibrational energy per unit surface. The power being injected into the plate $i$ is $P_{k}^{\text {inj }}=\sum_{s} \rho_{\alpha}\left(S_{s}\right)$ where the sum runs over all source points $S_{s}$ of the plate $i$. The usual asymptotic value is adopted for an estimation of the modal density $n_{k}=A_{k} \omega / 2 \pi c_{\phi_{k}} c_{k}$ where $c_{\phi_{k}}$ is the phase velocity for subsystem $k$. The modal overlap is then $m_{k}=\eta \omega n_{k}$. In the framework of SEA, we also need to introduce the coupling loss factors $\eta_{k l}$. The power being exchanged between subsystems $k=i, \alpha$ and $l=j, \beta$ is:

$$
P_{k l}=\omega\left(\eta_{k l} n_{k} T_{k}-\eta_{l k} n_{l} T_{l}\right)
$$

where $T_{k}=\bar{W}_{k} A_{k} / n_{k}$ is the modal energy sometimes called vibrational temperature. Many relationships may be found in the literature for $\eta_{k l}$. The one that we adopted here is based on a wave approach. It yields [1]:

$$
\eta_{k l}=\frac{L c_{k}}{\pi \omega A_{k}} \int_{0}^{\pi / 2} R_{i j, \alpha \beta}(\theta) \cos \theta d \theta
$$

where $L$ is the length of the coupling of plates $i$ and $j$. This relationship shows that the coupling loss factors verify the reciprocity relationship:

$$
\eta_{k l} n_{k}=\eta_{l k} n_{l}
$$

The SEA system is:

$$
\omega\left(\begin{array}{ccc}
n_{1} \sum_{m} \eta_{1 m} & & -n_{l} \eta_{l k} \\
& \ddots & \\
-n_{k} \eta_{k l} & & n_{N} \sum_{m} \eta_{N m}
\end{array}\right)\left(\begin{array}{c}
T_{1} \\
\vdots \\
T_{N}
\end{array}\right)=\left(\begin{array}{c}
P_{1}^{\mathrm{inj}} \\
\vdots \\
P_{N}^{\mathrm{inj}}
\end{array}\right)
$$

where $\eta_{k k}$ denotes the damping loss factor of the subsystem $k$. This system is symmetric by virtue of the reciprocity relationship.

The next section is devoted to the comparison of some results of the software CeReS and SEA with some measurements achieved on two structures. 


\section{Experimental results}

Two experiments have been performed on structures. Both are in steady state condition. The principle of these measurements is as follows. One or two shakers type B\&K4809 apply a force to the structure. The excitation signal is a white noise confined into the frequency band of interest. In the case of several shakers, their respective signals are uncorrelated. An impedance head type B\&K8001 is located between the shaker and the structure. It allows the measurement of the RMScross-spectrum force-acceleration $S_{f a}(\omega)$ for each driving point. All transducer signals are acquired with a FFT-analyzer type HP3665. The power being injected in flexural waves into a wide band was determined with a frequency-integration of the cross-spectrum $\rho_{b}^{s}=\Re \int_{\omega_{1}}^{\omega_{2}} S_{f a}(\omega) /(i \omega) d \omega$. No power is supplied to the other kinds of waves and thus, $\rho_{\alpha}^{s}=0$ for $\alpha=l, t$. For a third octave band, $\omega_{1}=\omega_{0} / 2^{1 / 6}$ and $\omega_{2}=\omega_{0} 2^{1 / 6}$ where $\omega_{0}$ is the centered frequency. The power spectral density $S_{v v}(\omega)$ of velocity is measured at several points with an accelerometer type B\&K4393V connected to an integrator amplifier type B\&K2635. The energy contained in the frequency band is determined from the power spectral density assuming that it is twice the kinetic energy. Thus $W_{\text {meas }}\left(\omega_{0}\right)=\rho \int_{\omega_{1}}^{\omega_{2}} S_{v v}(\omega) d \omega$ where $\rho$ is the mass per unit area of the plate. This measurement will be compared with the energy $W_{\text {CeReS }}$ provided by CeReS and the mean energy $\bar{W}_{\text {SEA }}$ of Statistical Energy Analysis.

The first experiment deals with a U-shaped aluminium plate with two slits. Figure 6a shows the experimental set-up and Figure 6b shows the geometry of the U-plate. The thickness is $1.5 \mathrm{~mm}$ and the plate is covered with a damping material in order to avoid a diffuse field. It is assumed that the presence of the damping material affects the damping loss factor and the surface mass density but not the bending rigidity of the aluminium plate. The surface mass density of the plate is $\rho=5.2 \mathrm{~kg} / \mathrm{m}^{2}$. The damping loss factor is $\eta=15 \%$ over all octave bands of interest and was measured on a piece of square plate (with the same damping material) excited by a shaker with a known injected power. The U-plate is excited by two shakers whose locations are shown in Figure 6b. The structure is tested over four octave bands from $400 \mathrm{~Hz}$ to $6400 \mathrm{~Hz}$. The values of injected powers, modal overlap and wavelength are summarized in Table 2. The power spectral density $S_{v v}(\omega)$ of velocity is measured at 22 points on a single line from top to bottom (see the measurement line in Figure 6b). The CeReS model acounts for flexural waves solely. Other kinds of waves cannot be created since the structure is flat. The boundary of the plate is divided into 70 elements. The CeReS model thus contains 70 degrees of freedom. The values of injected powers of Table 2 and the damping loss factor are put in data files of CeReS. The CPU-time is $24 \mathrm{~s}$ for twelve frequencies on a computer HP with a processor HPPA8500. SEA calculation is trivial in this case since there is a unique subsystem. The mean energy is simply $\bar{W}_{\mathrm{SEA}}=\left(\rho_{b}^{1}+\rho_{b}^{2}\right) / A \eta \omega$ where $A=0.801 \mathrm{~m}^{2}$ is the area of the U-plate. Figures $7 \mathrm{a}$ and $7 \mathrm{~b}$ shows some comparisons of the measured energy $\rho S_{v v}(\omega)$ in pure tone with the predicted values $W_{\text {CeReS }}$ versus frequency at points 14 and 21. The modal overlap is high. The variations of the pure tone response are due to the modal behavior of the structure and the CeReS prediction should be considered as RMS-value of energy in wide-band. However, the macroscopic evolution is well-predicted. In particular, the difference of vibrational energy between points 14 and 21 is $15.6 \mathrm{~dB}$ at $898 \mathrm{~Hz}$ and increases to $23.7 \mathrm{~dB}$ at $3592 \mathrm{~Hz}$. These points are mainly affected by the second shaker. Point 14 is at a distance $r_{1}=18 \mathrm{~cm}$ of this shaker whereas point 21 is at $r_{2}=72 \mathrm{~cm}$. On the other hand, the attenuation factor $m=\eta \omega / c$ is $m_{1}=3.9 \mathrm{~m}^{-1}$ at $898 \mathrm{~Hz}$ and $m_{2}=7.9 \mathrm{~m}^{-1}$ at $3592 \mathrm{~Hz}$. If we consider that both points are in the direct field $e^{-m r} / 2 \pi r$ of the second source, the difference of level may be evaluated with $10 \mathrm{~m}\left(r_{2}-r_{1}\right) \log _{10}(e)+10 \log _{10}\left(r_{2} / r_{1}\right)$ that is $15 \mathrm{~dB}$ at $898 \mathrm{~Hz}$ and $24.5 \mathrm{~dB}$ at $3592 \mathrm{~Hz}$. The increase of the difference can be explained with the increase of the attenuation. Figures $7 \mathrm{c}$ and $7 \mathrm{~d}$ show some comparisons of the measured energy $W_{\text {meas }}$ in third 
(a)

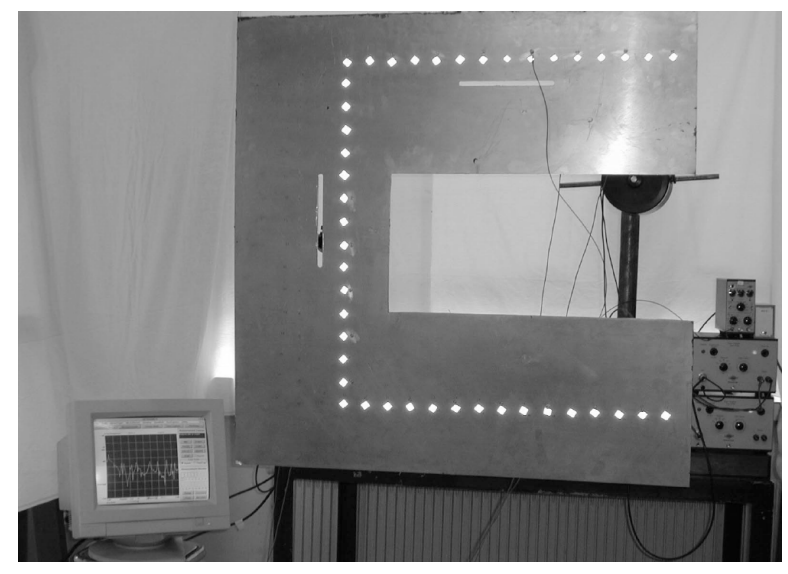

(b)

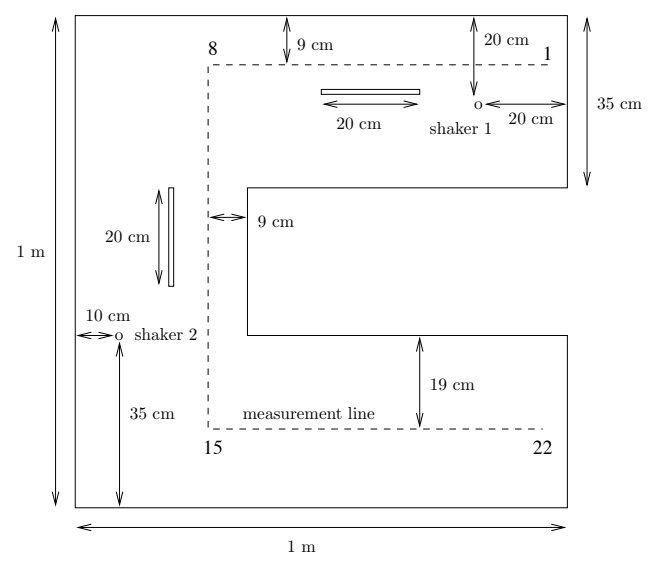

Figure 6: Experiment on a U-shaped plate. (a) View of the experimental setup. (b) Geometry of the U-plate and position of the two shakers, the two slits and the measurement line (points 1 to $22)$.

octave band and the predictions $W_{\text {CeReS }}$ and $\bar{W}_{\text {SEA }}$ along the measurement line (points 1 to 22 ). The two main peaks result from the proximity of point source while the secondary peak predicted by CeReS (points 9, 10) stems from the fact that the measurement line re-enter into the direct field of shaker 1. Unfortunately, this effect is lower than the effect of some dominant modes and cannot be observed. The mean energy $\bar{W}_{\mathrm{SEA}}$ seems to be over-estimated. But in fact, the mean level along the measurement line is not the mean level over the plate and there is a difference of $? \mathrm{~dB}$ at $1796 \mathrm{~Hz}$ and ? dB at $4525 \mathrm{~Hz}$. The mean level $\bar{W}_{\mathrm{SEA}}$ is correct compared with the mean level of the plate. This simply means that the damping loss factor has been correctly estimated.

The second experiment is concerned with a more complex structure. Figure 8a shows the experimental setup and Figure 8b the geometry of this structure. It is made of seven plates of steel. The thickness is $0.8 \mathrm{~mm}$ and the structure is entirely covered with a damping material named CATANE AL with thickness $1.24 \mathrm{~mm}$ and density $1.26 \mathrm{~g} / \mathrm{cm}^{3}$. An equivalent surface mass density 


\begin{tabular}{|c||c|c||c|c|}
\hline frequency & modal overlap & wavelength & power 1 & power 2 \\
\hline \hline $449 \mathrm{~Hz}$ & 13 & $16 \mathrm{~cm}$ & $34 \mu W$ & $19 \mu W$ \\
\hline $566 \mathrm{~Hz}$ & 17 & $15 \mathrm{~cm}$ & $19 \mu W$ & $10 \mu W$ \\
\hline $713 \mathrm{~Hz}$ & 21 & $13 \mathrm{~cm}$ & $12 \mu W$ & $9.3 \mu W$ \\
\hline $898 \mathrm{~Hz}$ & 26 & $12 \mathrm{~cm}$ & $9.6 \mu W$ & $6.2 \mu W$ \\
\hline $1131 \mathrm{~Hz}$ & 33 & $11 \mathrm{~cm}$ & $6.1 \mu W$ & $2.7 \mu W$ \\
\hline $1425 \mathrm{~Hz}$ & 42 & $9.5 \mathrm{~cm}$ & $4.5 \mu W$ & $2.4 \mu W$ \\
\hline $1796 \mathrm{~Hz}$ & 53 & $8.4 \mathrm{~cm}$ & $6.2 \mu W$ & $3 \mu W$ \\
\hline $2263 \mathrm{~Hz}$ & 67 & $7.5 \mathrm{~cm}$ & $13 \mu W$ & $6.9 \mu W$ \\
\hline $2851 \mathrm{~Hz}$ & 84 & $6.7 \mathrm{~cm}$ & $23 \mu W$ & $8.6 \mu W$ \\
\hline $3592 \mathrm{~Hz}$ & 106 & $6.0 \mathrm{~cm}$ & $0.8 \mu W$ & $0.4 \mu W$ \\
\hline $4525 \mathrm{~Hz}$ & 133 & $5.3 \mathrm{~cm}$ & $0.1 \mu W$ & $0.07 \mu W$ \\
\hline $5702 \mathrm{~Hz}$ & 168 & $4.7 \mathrm{~cm}$ & $0.05 \mu W$ & $0.02 \mu W$ \\
\hline
\end{tabular}

Table 2: Modal overlap, wavelength and powers being injected in the U-plate structure for each third octave band.

(a)

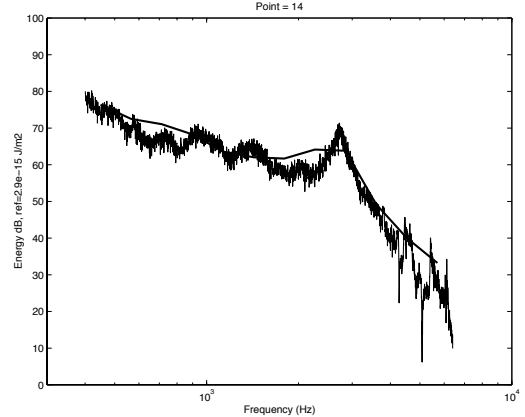

(c)

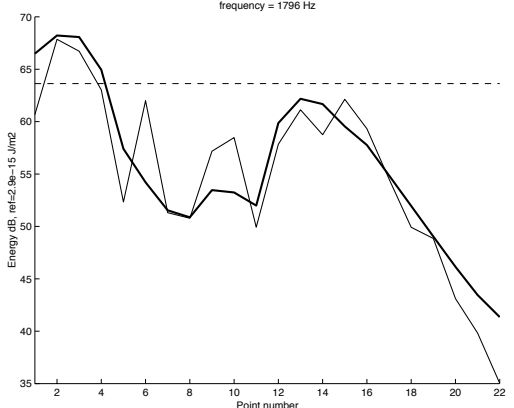

(b)

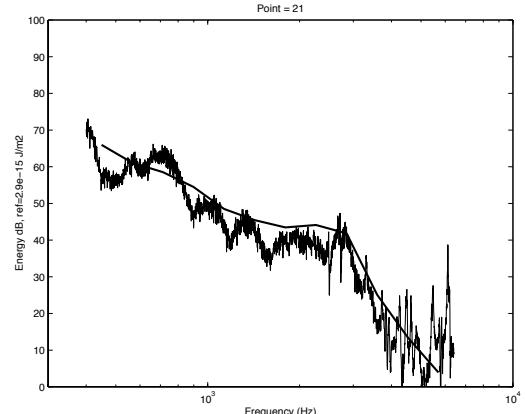

(d)

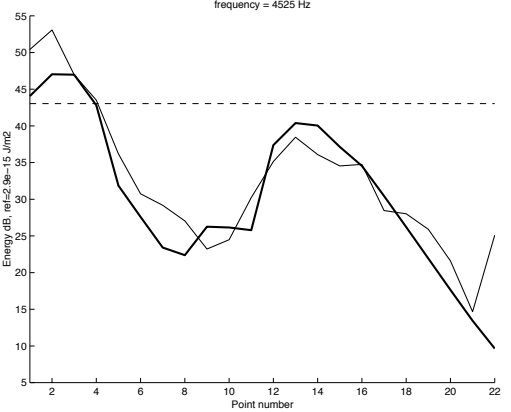

Figure 7: U-shaped plate. Comparison of measured energy in pure tone (thin line) and predicted RMS-values (thick line) by the software CeReS versus frequency at (a) point 14, (b) point 21. Comparison of measured mean values of the energy density (thin line); SEA values (broken line) and predicted values by the software CeReS (thick line) for the third-octave bands centered at (c) $1796 \mathrm{~Hz}$ and (d) $4525 \mathrm{~Hz}$. 


\begin{tabular}{|c||c|c||c|}
\hline frequency & modal overlap & wavelength & power \\
\hline \hline $449 \mathrm{~Hz}$ & 1.3 & $12.5 \mathrm{~cm}$ & $8.5 \mu W$ \\
\hline $566 \mathrm{~Hz}$ & 1.6 & $11.2 \mathrm{~cm}$ & $3.5 \mu W$ \\
\hline $713 \mathrm{~Hz}$ & 2.0 & $10 \mathrm{~cm}$ & $4.0 \mu W$ \\
\hline $898 \mathrm{~Hz}$ & 2.5 & $9 \mathrm{~cm}$ & $1.5 \mu W$ \\
\hline $1131 \mathrm{~Hz}$ & 3.2 & $7.9 \mathrm{~cm}$ & $1.3 \mu W$ \\
\hline $1425 \mathrm{~Hz}$ & 4.0 & $7 \mathrm{~cm}$ & $0.7 \mu W$ \\
\hline $1796 \mathrm{~Hz}$ & 5.0 & $6.3 \mathrm{~cm}$ & $0.9 \mu W$ \\
\hline $2263 \mathrm{~Hz}$ & 6.4 & $5.6 \mathrm{~cm}$ & $0.4 \mu W$ \\
\hline $2851 \mathrm{~Hz}$ & 8.0 & $5 \mathrm{~cm}$ & $0.8 \mu W$ \\
\hline $3592 \mathrm{~Hz}$ & 10.1 & $4.4 \mathrm{~cm}$ & $1.4 \mu W$ \\
\hline $4525 \mathrm{~Hz}$ & 12.7 & $4 \mathrm{~cm}$ & $2.3 \mu W$ \\
\hline $5702 \mathrm{~Hz}$ & 16.0 & $3.5 \mathrm{~cm}$ & $1.0 \mu W$ \\
\hline $7184 \mathrm{~Hz}$ & 20.2 & $3.1 \mathrm{~cm}$ & $1.1 \mu W$ \\
\hline
\end{tabular}

Table 3: Modal overlap, wavelength and power being injected in the seven-plate structure for each third octave band.

is used, assuming again that the presence of the damping material does not affect the rigidity of steel plates. The surface mass density is $\rho=7.8 \mathrm{~kg} / \mathrm{m}^{2}$ and the loss factor is $\eta=2 \%$. The same technique of measurement was involved. The frequency bands of interest cover from $400 \mathrm{~Hz}$ to $12800 \mathrm{~Hz}$. However measurement was poor in the last two third octave bands and thus results are presented from $400 \mathrm{~Hz}$ to $8000 \mathrm{~Hz}$. The CeReS model of this structure takes into account three kinds of wave, and the structure was discretized with 200 boundary elements. Thus the CeReS model contains 600 degrees of freedom. Values of injected power, wavelength and modal overlap are summarized in Table 3. The required CPU-time for this model is $3347 \mathrm{~s}$ for fifteen frequencies on the same computer. But, if we neglect in-planes waves, the model reduces to 200 degrees of freedom and CPU-time becomes $318 \mathrm{~s}$. No significant difference has been observed on results. Figures $9 \mathrm{a}, 9 \mathrm{~b}$ and $9 \mathrm{c}$ show some comparisons of measured energy $\rho S_{v v}(\omega)$ in pure tone with the

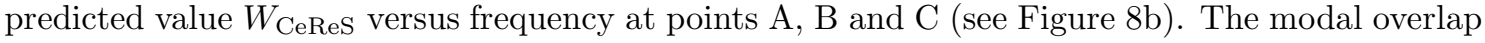
is lower than for U-plate. Figure $9 \mathrm{~d}$ show a comparison of the measured energy $W_{\text {meas }}$ in the frequency band $6400-8000 \mathrm{~Hz}$, the prediction $W_{\mathrm{CeReS}}$ of the software CeReS and the mean energy $\bar{W}_{\text {SEA }}$ along the measurement line drawn in Figure $8 \mathrm{~b}$. Within the central plate (points 7 to 18) the total energy is well predicted by both CeReS and SEA models. In the neighborhood of the driving point (points 7 to 12), CeReS prediction shows a sharp peak because the direct field is singular, like $1 / r$ where $r$ is the source-receiver distance. Indeed this singularity has no physical meaning and the measurement clearly shows that this model the model is not correct in near-field say, within one wavelength. However, the prediction is correct for points 13 to 18 where the decrease is well-predicted. The decrease of energy inside other plates is more difficult to observe. These lateral plates are smaller than the central one and the decrease is lower. But the step of energy from the central plate to lateral ones is well-predicted. It seems that SEA slightly under-estimates the energy in the first plate (points 1 to 6 ) but measurements in other frequencies do not comfirm it. The last plate (points 19 to 23 ) is clearly to narrow and it is difficult to conclude anything. The width is $4 \mathrm{~cm}$ and the wavelength $3 \mathrm{~cm}$ at $7100 \mathrm{~Hz}$. The high frequency assumption does not apply and it should be better to consider it as a beam. 
(a)

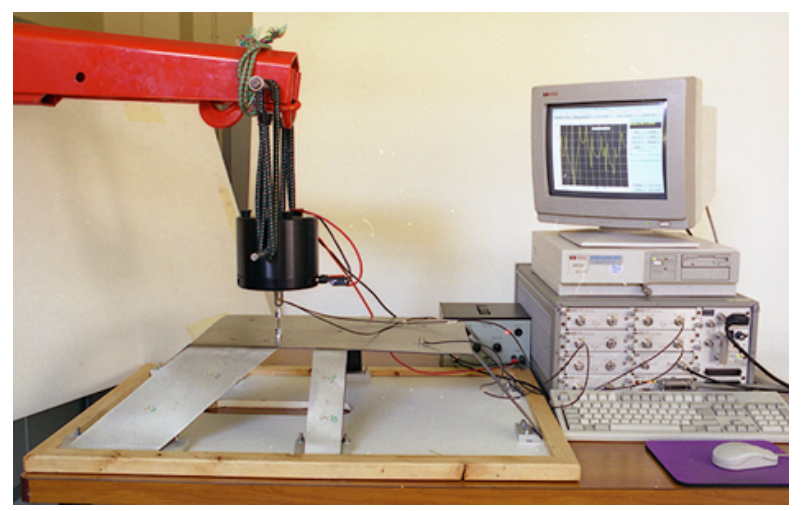

(b)

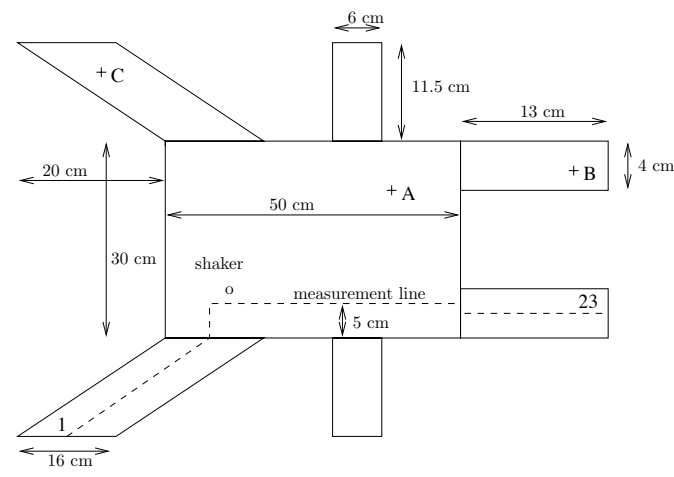

Figure 8: Experiment on a seven-plates structure. (a) View of the experimental setup. (b) Geometry of the structure and position of the shaker and measurement points A, B, C and the measurement line (points 1 to 23 ). 
(a)

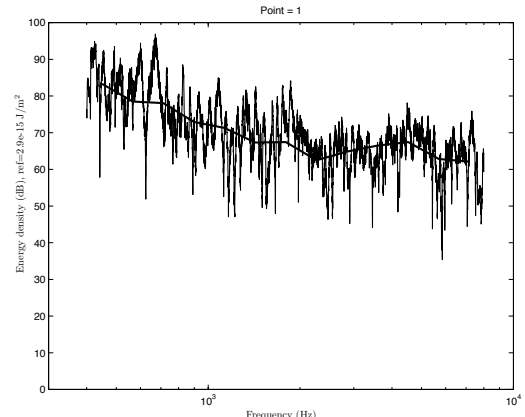

(c)

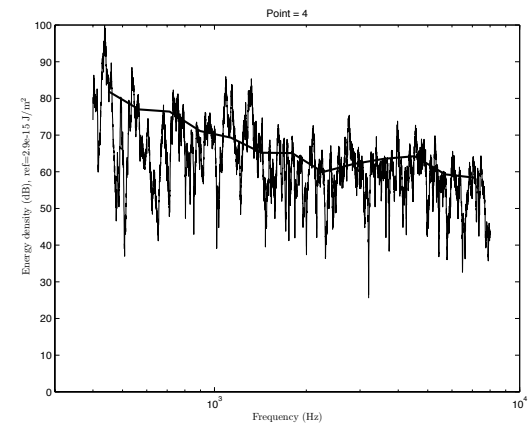

(b)

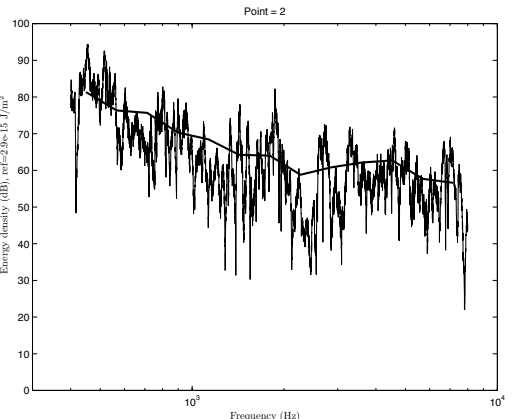

(d)

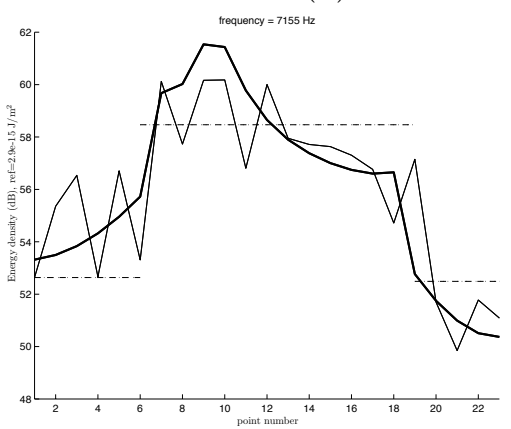

Figure 9: Seven-plates structure. Comparison of measured energy in pure tone (thin line) and predicted RMS-values (thick line) by the software CeReS versus frequency at (a) point A, (b) point B, (c) point C. (d) Comparison of measured mean values over the third octave-band centered at $7155 \mathrm{~Hz}$ of energy (thin line); SEA values (broken line) and CeReS values (thick line) versus position (points 1 to 23 ). 


\section{Conclusion}

In this paper, we have derived the equations for the vibrational energy transfer in structures. These equations have been obtained under the high frequency assumption that at least several wavelengths lie in the propagation domain and, when time-variation is accounted for, the period of the underlying wave-packets is small compared with the characteristic time of the overall phenomenon.

It has been found that this model is analogous to the problem of radiative heat transfer. Although some differences exist between heat and mechanical transfer, it may be possible to use a thermal sofware in vibroacoustics. In the past, it has ever been attempted to re-use thermal softwares in dynamics [3]. But it was based on an analogy with a conduction problem. Radiative transfer differs from the conduction and so, although the vibrational conductivity approach and the present approach are both based on a thermal analogy, they are not equivalent.

The sofware CeReS is especially designed for the solving of the integral equations presented in this paper. The numerical scheme chosen is rather classical. Any other scheme would be suitable. An important fact is that the kernel of this integral equation is regular. This greatly simplify the choice of quadrature scheme.

Numerical results provided by CeReS, SEA and measurements achieved on two multi-plate structures, are in good agreement. They highlight that the present method is a natural extent of SEA since it provides the repartition of energy inside subsystems. Indeed this gain of information has a counterpart. CeReS requires more CPU-time than SEA, but fortunately, significantly less than a FEM model for these frequencies.

\section{Acknowledgments}

The author gratefully acknowledges the Région Rhône-Alpes for the financial support.

\section{Appendix A: Gaussian wave packet}

Let denote $v_{\alpha}(x, t) \alpha=b, l$ or $t$ the disturbance in space-time for vibration in plates, assumed to depend on a single space variable $x$ for the sake of simplicity. Therefore we consider a onedimensional system, a beam, or alternatively a plane wave traveling in a two-dimensional system, a plate. Anyway the spreading occuring in multi-dimensional systems is not taken into account. A convenient choice is the transverse deflection for $v_{b}$ and the longitudinal and transverse potentials $v_{l}$ and $v_{t}$ for in-plane motion. Using a spatial Fourier transform:

$$
V_{\alpha}(k, t)=\frac{1}{2 \pi} \int_{-\infty}^{\infty} v_{\alpha}(x, t) e^{i k x} d x
$$

it is straighforward to see that $V_{\alpha}(k, t)=V_{\alpha}(k, 0) e^{i \omega t}$ is solution of a second order differential equation respect to time, where $\omega$ is constrained to verify the dispersion relationship.

$$
\begin{array}{ll}
c_{\phi_{\alpha}}^{2} k^{2}-\omega^{2}=0 & \text { for } \quad \alpha=l \text { or } t \\
D k^{4}-\rho \omega^{2}=0 & \text { for } \quad \alpha=b
\end{array}
$$


Recall that $D$ is the bending stiffness and $\rho$ the mass per unit area. By using the inverse Fourier transform $v_{\alpha}(x, t)$ may be synthesized by the superposition plane waves traveling in the positive $x$-direction for instance.

$$
v_{\alpha}(x, t)=\int_{-\infty}^{\infty} V_{\alpha}(k, 0) e^{i(\omega t-k x)} d k
$$

A gaussian wave packet at initial time has the shape:

$$
v_{\alpha}(x, 0)=A e^{-\frac{x^{2}}{4 \sigma_{0}^{2}}} e^{-i k_{0} x}
$$

where $A$ is the amplitude of the disturbance, $\sigma_{0}$ the spatial spread of the packet and $k_{0}$ the wavenumber of the main oscillation. The high frequency assumption then states that the spatial spread $\sigma_{0}$ is much greater than the wavelength $2 \pi / k_{0}$. Applied to the function (47), the Fourier transform $V_{\alpha}(k, 0)$ becomes

$$
V_{\alpha}(k, 0)=\frac{A \sigma_{0}}{\sqrt{\pi}} e^{-\sigma_{0}^{2}\left(k-k_{0}\right)^{2}}
$$

For the next step, it is convenient to re-write the dispersion relationship (45) as

$$
\omega=\omega_{0}+c_{\alpha}^{0}\left(k-k_{0}\right)+d_{\alpha}\left(k-k_{0}\right)^{2}
$$

where $\omega_{0}=k_{0} c_{\phi_{\alpha}}^{0}$ is the circular frequency corresponding to the wavenumber $k_{0}, c_{\phi_{\alpha}}^{0}, c_{\alpha}^{0}$ respectively the phase and group velocities in same condition and $d_{\alpha}=0$ for $\alpha=l$ or $t$ and $d_{b}=\sqrt{D / \rho}$. Now the disturbance at any time $t$ is obtained from the Fourier integral (46) where (47) and (49) have been substituted, with the result [21]

$$
v_{\alpha}(x, t)=A \frac{\sigma_{0}}{\sigma(t)} e^{-\frac{\left(x-c_{\alpha} t\right)^{2}}{4 \sigma(t)^{2}}} e^{i\left(\omega_{0} t-k_{0} x\right)}
$$

where the spatial spread at time $t$ is given by

$$
\sigma(t)^{2}=-\sigma_{0}^{2}+i d_{\alpha} t
$$

It is then clear that initial gaussian wave packet remains a gaussian wave packet at later time with an increasing spatial spread and so that the high frequency assumption tends to be better verified. Furthermore, the high frequency assumption implies that space-time derivatives of the gaussian shape function $\frac{\sigma_{0}}{\sigma(t)} e^{-\frac{\left(x-c_{(t)}\right)^{2}}{4 \sigma(t)^{2}}}$ are small compared with similar derivatives of the oscillatory term $e^{i\left(\omega_{0} t-k_{0} x\right)}$. This results in considerable simplifications when evaluating energy quantities. For instance, consider $T(x, t)$ the kinetic energy density and $V(x, t)$ the potential energy density for out-of-plane motion of plates.

$$
\begin{gathered}
T(x, t)=\frac{1}{2} \rho\left|\frac{\partial v_{b}}{\partial t}\right|^{2}=\frac{\rho \omega_{0}^{2}}{2}\left|v_{b}\right|^{2} \\
V(x, t)=\frac{1}{2} D\left|\frac{\partial^{2} v_{b}}{\partial x^{2}}\right|^{2}=\frac{D k_{0}^{4}}{2}\left|v_{b}\right|^{2}
\end{gathered}
$$

where the $y$-derivative terms in potential energy of plates has been removed. The equality

$$
T(x, t)=V(x, t)
$$

is established and the total energy density is

$$
W(x, t)=T(x, t)+V(x, t)=\rho \omega_{0}^{2}\left|v_{b}\right|^{2}
$$


Let remark that Equation (54) is only valid for one wave packet or one traveling wave. But indeed, it does not apply in general case with more waves due to interference phenomena. However, it is surprising that it is still valid in farfield for two traveling waves in a beam although this remark is of no importance for the purpose of this paper. Further, consider the energy flow $I(x, t)$

$$
I(x, t)=D\left(\frac{\partial^{2} v_{b}}{\partial x^{2}} \frac{\partial^{2} v_{b}^{*}}{\partial x \partial t}-\frac{\partial^{3} v_{b}}{\partial x^{3}} \frac{\partial v_{b}^{*}}{\partial t}\right)=2 D k_{0}^{3} \omega_{0}\left|v_{b}\right|^{2}
$$

The proportionality constant between $I(x, t)$ and $W(x, t)$ is

$$
2 D k_{0}^{3} \omega_{0} / \rho \omega_{0}^{2}=2 \frac{D}{\rho \omega_{0}^{2}} \omega_{0} k_{0}^{3}=2 \frac{1}{k_{0}^{4}} \omega_{0} k_{0}^{3}=2 \frac{\omega_{0}}{k_{0}}=c_{b}
$$

and the equality

$$
I(x, t)=c_{b} W(x, t)
$$

is thus demonstrated. Equalities (54) and (58) remain valid for in-plane motions.

\section{Appendix B: Power balance for direct fields}

This Appendix is intended to verify equality (5) where functions $G_{\alpha}$ and $\mathbf{H}_{\alpha}$ are defined in (6-9). Since the subscript $\alpha$ does not matter in this proof, it is temporarily removed. For the sake of clarity, the source point $S$ and the initial time $\tau$ are removed from the parenthesis and $G(S, \tau ; M, t)$ and $G(S, M)$ are rather noted $G_{S, \tau}(M, t)$ and $G_{S}(M)$. Indeed $G_{S, \tau}$ and $\mathbf{H}_{S, \tau}$ must be considered as generalized functions and the first step is to seek a mathematical sense for these symbols.

The function $G_{S}$ of variable $M$ is locally integrable $\left(\in L_{l o c}^{1}\left(\mathbb{R}^{n}\right)\right.$ where $n=1,2$ or 3$)$ so that $G_{S, \tau}$ is the product of a dirac function and that function. Consider a test function $\varphi \in C_{c}^{\infty}\left(\mathbb{R}^{n+1}\right)$ whose support is compact, the formal calculation

$$
<G_{S, \tau}, \varphi>=\int_{\mathbb{R}^{n+1}} \delta_{\tau^{\prime}}(t) G_{S}(M) \varphi(M, t) d M d t=\int_{\mathbb{R}^{n}} G_{S}(M) \varphi\left(M, \tau^{\prime}\right) d M
$$

where $\tau^{\prime}=\tau+S M / c$ suggests that a correct definition for the generalized function $G_{S, \tau}$ should be the right-hand side of (59). The inequality $\left|\left\langle G_{S, \tau}, \varphi\right\rangle\right| \leq\|\varphi\|_{\infty} \int_{K} G_{S}(M) d M$ where $\varphi(M, t)=0$ whenever $M \notin K$ compact, shows that the last integral of (59) makes sense and that the linear map $\varphi \mapsto<G_{S, \tau}, \varphi>$ is continuous for the usual topology of $C_{c}^{\infty}\left(\mathbb{R}^{n+1}\right)$ and has order 0 . It is therefore a distribution. $\mathbf{H}_{S, \tau}$ is defined in the same manner.

Our purpose is to evaluate the distribution $R_{S, \tau}=\nabla \cdot \mathbf{H}_{S, \tau}+m c G_{S, \tau}+\frac{\partial}{\partial t} G_{S, \tau}$. To this end, consider a test function $\varphi$ whose compact support does not contain $(S, \tau)$.

$$
\begin{aligned}
& <R_{S, \tau}, \varphi>= \\
& \int_{\mathbb{R}^{n}}-\mathbf{H}_{S}(M) \cdot(\nabla \varphi)\left(M, \tau^{\prime}\right)+m c G_{S}(M) \varphi\left(M, \tau^{\prime}\right)-G_{S}(M) \frac{\partial \varphi}{\partial t}\left(M, \tau^{\prime}\right) d M
\end{aligned}
$$

Since $\varphi(M, t)=0$ for $S M<\epsilon$ and $|t-\tau|<c \epsilon$ where $\epsilon$ is sufficiently small, the latter integral may be evaluated over $S M \geq \epsilon$. The relationship

$$
(\nabla \varphi)\left(M, \tau^{\prime}\right)=\nabla\left[\varphi\left(M, \tau^{\prime}\right)\right]-\frac{1}{c} \frac{\partial \varphi}{\partial t}\left(M, \tau^{\prime}\right) \mathbf{u}_{S M}
$$


yields

$$
\begin{aligned}
<R_{S, \tau}, \varphi> & =\int_{S M \geq \epsilon}-\mathbf{H}_{S}(M) \cdot \nabla\left[\varphi\left(M, \tau^{\prime}\right)\right]+m c G_{S}(M) \varphi\left(M, \tau^{\prime}\right) d M \\
& =\int_{S M \geq \epsilon}\left[\nabla \cdot \mathbf{H}_{S}(M)+m c G_{S}(M)\right] \varphi\left(M, \tau^{\prime}\right) d M \\
& =0
\end{aligned}
$$

where the second integral has been obtained by integration by parts and the last equality results from the function equality $\nabla . \mathbf{H}_{S}+m c G_{S}=0$ on $\mathbb{R}^{n}-\{S\}$ which may be checked by a direct calculation. The support of the distribution $R_{S, \tau}$ is $\{(S, \tau)\}$ and $R_{S, \tau}$ is therefore a sum of derivatives of $\delta_{S, \tau}$ up to the order of $R_{S, \tau}$ that is one.

Consider a test function $\varphi(r, t)$ depending on the distance $r=S M$. Adopting polar coordinates for such a function

$$
\begin{aligned}
<R_{S, \tau}, \varphi> & =\gamma_{0} \int_{0}^{\infty}-H(r) \frac{\partial \varphi}{\partial r}\left(r, \tau^{\prime}\right)+m c G(r) \varphi\left(r, \tau^{\prime}\right)-G(r) \frac{\partial \varphi}{\partial t}\left(r, \tau^{\prime}\right) r^{n-1} d r \\
& =\int_{0}^{\infty}-e^{-m r} \frac{\partial}{\partial r}\left[\varphi\left(r, \tau^{\prime}\right)\right]+m e^{-m r} \varphi\left(r, \tau^{\prime}\right) d r \\
& =\left[-e^{-m r} \varphi\left(r, \tau^{\prime}\right)\right]_{r=0}^{r=\infty} \\
& =\varphi(0, \tau)
\end{aligned}
$$

and we conclude that

$$
R_{S, \tau}=\nabla \cdot \mathbf{H}_{S, \tau}+m c G_{S, \tau}+\frac{\partial}{\partial t} G_{S, \tau}=\delta_{S, \tau}
$$

\section{Appendix C: Reflection efficiencies}

This appendix is intended to derive the relationships for the reflection efficiencies $R_{\beta \alpha}\left(\theta_{\beta}\right)$ defined in (20). These efficiencies just depend on the incident angle $\theta_{\beta}$. The knowledge of these reflection efficiencies is necessary for a practical solving of the integral equation (23). So, we are interested in the conversion mode phenomenon that occurs when a wave of type $\beta=b, l$ or $t$ impinges on a free, clamped or simply supported edge of a Love plate.

When a wave-packet impinges on a boundary the interaction process duration is much greater than the period of the main oscillation. It has also been assumed that the time-variation of the shape function is much slower than the one of the main oscillation. It results that the behavior of wave-packets and traveling waves are quite similar during the interaction process. In addition, when the boundary is locally flat, the locality principle allows us to substitute an infinitely extended straight boundary for the actual boundary. Let add that only polygonal boundaries are included in the software CeReS. That leads us to study the canonical problem of an incident traveling plane wave propagating toward a straight edge of a semi-infinite plate. This problem has been largely commented in literature, see for instance Reference [22], and we shall confine the present discussion to energetic aspect. It should be added that more complicated canonical problems with curved edges or curved wavefronts are solved in Reference [23] in electromagnetics. Other related references are also included.

First, when an incident flexural wave responsible of out-of-plane motion $v$ is considered two waves are reflected. The first is a flexural traveling wave whereas the second is an evanescent wave. It has ever been remarked that no energy flow is associated to any evanescent wave. The reflected flux is thus totally carried by the reflected traveling wave. In addition, free, clamped and simply supported edges are all non-absorbing boundaries. It results that:

$$
R_{b \alpha}\left(\theta_{\beta}\right)=0 \quad \alpha=l, t \quad R_{b b}\left(\theta_{\beta}\right)=1
$$




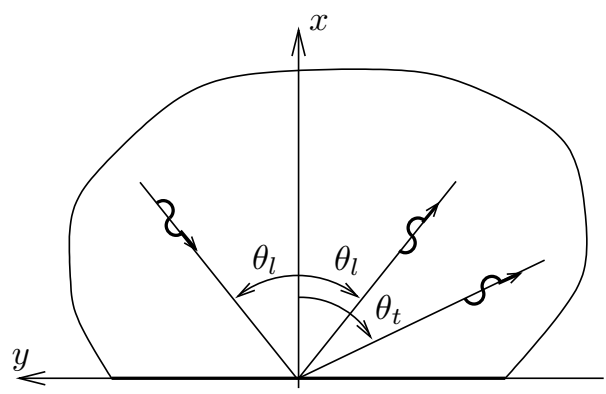

Figure 10: Two waves of types $\beta=l$ and $t$ are reflected when a longitudinal wave impinges on a free edge.

And applying the reciprocity condition

$$
R_{\alpha b}\left(\theta_{\beta}\right)=0 \quad \alpha=l, t
$$

Second, when the incident wave type is $\beta=l$ or $t$ the in-plane motion is reflected into two waves of type $\alpha=l$ and $t$ (see Figure 10). No out-of-plane motion is created. Then by denoting $\varphi$ and $\psi$ the potentials associated to the longitudinal and the transverse waves, incident the sums of incident and reflected waves are written:

$$
\begin{aligned}
& \varphi(x, y)=\delta_{\beta l} e^{i\left(\omega t+k_{l, x} x+k_{l, y} y\right)}+a_{\beta l} e^{i\left(\omega t-k_{l, x} x+k_{l, y} y\right)} \\
& \psi(x, y)=\delta_{\beta t} e^{i\left(\omega t+k_{t, x} x+k_{t, y} y\right)}+a_{\beta t} t e^{i\left(\omega t-k_{t, x} x+k_{t, y} y\right)}
\end{aligned}
$$

The first terms in the right-hand side are for incident waves, $\delta_{\beta l}=1$ and $\delta_{\beta t}=0$ for an incident longitudinal wave and $\delta_{\beta l}=0$ and $\delta_{\beta t}=1$ for an incident transverse wave. The reflected plane waves (second terms in the right-hand side) are obtained by reversing the sign of $k_{\beta, x}$. We have noted

$$
k_{\beta, x}=\frac{\omega}{c_{\phi_{\beta}}} \cos \theta_{\beta} \quad k_{\beta, y}=\frac{\omega}{c_{\phi_{\beta}}} \sin \theta_{\beta}
$$

the wavenumber components and $c_{\phi_{\beta}}$ the phase velocities. The angles $\theta_{l}$ and $\theta_{t}$ are related by the Snell law $\sin \theta_{t} / c_{\phi_{t}}=\sin \theta_{l} / c_{\phi_{l}}$ Transverse waves always propagate slower than longitudinal waves and so the Snell shows that in case of an incident transverse wave the angle $\theta_{l}$ is defined when $\theta_{t} \leq \arcsin c_{\phi_{t}} / c_{\phi_{l}}$. For greater values of $\theta_{t}$ the reflected longitudinal wave is evanescent. The case of an incident longitudinal wave does not reveal such a problem. The $x$-displacement noted $u$ and the $y$-displacement noted $v$ are related to the potentials with the relationships

$$
\begin{aligned}
& u=\frac{\partial \varphi}{\partial x}+\frac{\partial \psi}{\partial y} \\
& v=\frac{\partial \varphi}{\partial y}-\frac{\partial \psi}{\partial x}
\end{aligned}
$$

Finally, considering a section normal to the $x$-direction, the normal stress $N$ and the transverse stress $T$ are given by:

$$
\begin{aligned}
N & =D_{l}\left[\frac{\partial^{2} \varphi}{\partial x^{2}}+\nu \frac{\partial^{2} \varphi}{\partial y^{2}}+(1-\nu) \frac{\partial^{2} \psi}{\partial x \partial y}\right] \\
T & =D_{t}\left[\frac{\partial^{2} \psi}{\partial y^{2}}-\frac{\partial^{2} \psi}{\partial x^{2}}+2 \frac{\partial^{2} \varphi}{\partial x \partial y}\right]
\end{aligned}
$$


where $D_{l}$ and $D_{t}$ are the longitudinal and transverse rigidity. Now, applying the relevant boundary conditions at the edge $x=0$, the coefficients $a_{\beta \alpha}$ of the reflected waves may be calculated. For instance, a free edge imposes that $N=0$ and $T=0$ and so,

$$
\left(\begin{array}{cc}
k_{l, x}^{2}+\nu k_{l, y}^{2} & (\nu-1) k_{t, x} k_{t, y} \\
-2 k_{l, x} k_{l, y} & k_{t, y}^{2}-k_{t, x}^{2}
\end{array}\right)\left(\begin{array}{c}
a_{\beta l} \\
a_{\beta t}
\end{array}\right)=\left(\begin{array}{cc}
-k_{l, x}^{2}-\nu k_{l, y}^{2} & (\nu-1) k_{t, x} k_{t, y} \\
-2 k_{l, x} k_{l, y} & -k_{t, y}^{2}+k_{t, x}^{2}
\end{array}\right)\left(\begin{array}{c}
\delta_{\beta l} \\
\delta_{\beta t}
\end{array}\right)
$$

A similar system is obtained for clamped edge $(u=v=0)$. Magnitudes of reflected waves are determined by solving this linear system. The incident flux for in-plane motion is calculated from

$$
\mathcal{P}_{\text {inc }}=D_{\beta}\left[\left(k_{\beta, x}^{2}+k_{\beta, y}^{2}\right) \mathcal{I} m\left(k_{\beta, x}\right)\right]
$$

wheras a similar relationship applies for the reflected flux of kind $\alpha$.

$$
\mathcal{P}_{\text {ref }}=D_{\alpha}\left[\left(k_{\alpha, x}^{2}+k_{\alpha, y}^{2}\right) \mathcal{I} m\left(k_{\alpha, x}\right)\right]\left|a_{\beta \alpha}\right|^{2}
$$

The reflection efficiencies are finally obtained by taking the ratio of reflected flux over incident flux.

$$
R_{\beta \alpha}\left(\theta_{\beta}\right)=\frac{D_{\alpha}\left[\left(k_{\alpha, x}^{2}+k_{\alpha, y}^{2}\right) \mathcal{I} m\left(k_{\alpha, x}\right)\right]\left|a_{\beta \alpha}\right|^{2}}{D_{\beta}\left[\left(k_{\beta, x}^{2}+k_{\beta, y}^{2}\right) \mathcal{I} m\left(k_{\beta, x}\right)\right]}
$$

\section{References}

[1] R.H. Lyon. Statistical Energy Analysis of Dynamical Systems: Theory and Application. Cambridge, Massachusetts, MIT Press, 1975.

[2] R.S. Langley. A wave intensity technique for the analysis of high frequency vibrations. Journal of Sound and Vibration, 159(3):483-502, 1992.

[3] D.J. Nefske and S.H. Sung. Power flow finite element analysis of dynamic systems: Basic theory and application to beams. NCA Publication, 3, 1987.

[4] J.C. Wohlever and R.J. Bernhard. Mechanical energy flow models of rods and beams. Journal of Sound and Vibration, 153:1-19, 1992.

[5] O.M. Bouthier and R.J. Bernhard. Simple models of energy flow in vibrating membranes. Journal of Sound and Vibration, 182:129-147, 1995.

[6] O.M. Bouthier and R.J. Bernhard. Simple models of energy flow in vibrating plates. Journal of Sound and Vibration, 182:149-164, 1995.

[7] R.S. Langley. On the vibrational conductivity approach to high frequency dynamics for twodimensional structural components. Journal of Sound and Vibration, 182:637-657, 1995.

[8] A. Carcaterra and A. Sestieri. Energy density equations and power flow in structures. Journal of Sound and Vibration, 188(2):269-282, 1995.

[9] A. Le Bot. Geometric diffusion of vibrational energy and comparison with the vibrational conductivity approach. Journal of Sound and Vibration, 212(4):637-647, 1998.

[10] A. Carcaterra and L. Adamo. Thermal analogy in wace energy transfer: theoretical and experimental analysis. Journal of Sound and Vibration, 226(2):253-284, 1999. 
[11] M. N. Ichchou, A. Le Bot, and L. Jezequel. A transient local energy approach as an alternative to transient sea: wave and telegraph equations. Journal of Sound and Vibration, 246(5):829$840,2001$.

[12] Soize C., Desanti A., and David J.M. Méthodes numériques en élasto-acoustique BF et MF. La recherche aérospatiale, 5:25-44, 1992. see Section 2.5.

[13] C. Steel and L. Taber. Comparison of WKB and finite difference calculation for a twodimensional cochlear model. Journal of Acoustical Society of America, 65(4), 1977.

[14] K. De Langhe. High frequency vibrations: contributions to experimental and computional SEA parameter identification techniques. Technical Report ISBN 90-7380 2-50-4, Katholieke Universiteit Leuven, 1996. see Section 2.6.

[15] H.G.D. Goyder and R.G. White. Vibrational power flow machines into built-up structures, part I: Introduction and approximate analyses of beam and plate-like foundations. Journal of Sound and Vibration, 68(1):59-75, 1980.

[16] H.G.D. Goyder and R.G. White. Vibrational power flow machines into built-up structures, part II: wave propagation and power flow in beam-stiffened plates. Journal of Sound and Vibration, 68(1):77-96, 1980.

[17] A. Le Bot. A vibroacoustic model for high frequency analysis. Journal of Sound and Vibration, 211(4):537-554, 1998

[18] A. Le Bot and A. Bocquillet. Comparison of an integral equation on energy and the ray-tracing technique for room acoustics. Journal of Acoustical Society of America, 108(4):1732-1740, 2000 .

[19] Beckmann Th. Wöhle W. and Schreckenbach H. Coupling loss factors for statistical energy analysis of sound transmission at rectangular slab joints, part. I. Journal of Sound and Vibration, 77(3):323-334, 1981.

[20] E. Anderson and other authors. Lapack user's guide. Society for Industrial and Applied Mathematics, third edition edition, 1999.

[21] W.C. Elmore and M.A. Heald. Physics of waves. Dover, 1969. see page 432.

[22] A. Bedford and D.S. Drumheller. Introduction to elastic wave propagation. Wiley, 1994. see Section 3.3 .

[23] Y.A. Kravtsov and Y.I. Orlov. Geometrical optics of inhomogeneous media. Springer-Verlag, 1990. see Section 3.2. 